\title{
Multiobjective Performance-based Designs in Fault Estimation and Isolation for Discrete-time Systems and its Application to Wind Turbines
}

\author{
Ester Sales-Setién ${ }^{1 \star}$ and Ignacio Peñarrocha-Alós ${ }^{1}$ \\ ${ }^{1}$ Department of Industrial Engineering and Design, Universitat Jaume I, Castellón, Spain \\ *esales@uji.es
}

\begin{abstract}
In this work, we develop a performance-based design of model-based observes and statistical-based decision mechanisms for achieving fault estimation and fault isolation in systems affected by unknown inputs and stochastic noises. First, through semidefinite programming, we design the observers considering different estimation performance indices as the covariance of the estimation errors, the fault tracking delays and the degree of decoupling from unknown inputs and from faults in other channels. Second, we perform a codesign of the observers and decision mechanisms for satisfying certain trade-off between different isolation performance indices: the false isolation rates, the isolation times and the minimum size of the isolable faults. Finally, we extend these results to a scheme based on a bank of observers for the case where multiple faults affect the system and isolability conditions are not verified. To show the effectiveness of the results, we apply these design strategies to a well-known benchmark of wind turbines which considers multiple faults and has explicit requirements over isolation times and false isolation rates.
\end{abstract}

Keywords: Fault estimation, fault diagnosis, multiobjective optimisation, wind turbines.

\section{Introduction}

The importance of the reliability and maintainability of systems has increased over the last decades. Hence, much effort has been devoted to developing fault detection and isolation (FDI) and fault tolerant control (FTC) strategies (Gao, Cecati, \& Ding, 2015). On the FTC framework, there are two possible approaches: active and passive FTC. The difference between them is that passive FTC is just an application of robust control that considers faults as uncertainties while active FTC relies on fault diagnosis outputs. The two main approaches regarding FDI are data-based and model-based techniques, see (Ding, 2014) and (Ding, 2008; J. Chen \& Patton, 2012), respectively. Broadly speaking, most FDI systems consist of residual generators and evaluators; however, research has shown that there are intrinsic difficulties in the use of residuals in active FTC due to the complexity derived from the reconstruction of the faults from the residuals. These reconstructions rely on discrete-event algorithms with complex decisions that entail delays and errors (Lan \& Patton, 2016; Cieslak, Efimov, \& Henry, 2015). Active FTC based directly on fault estimation (FE) rather than on FDI seems to provide more immediate and accurate results, see (Lan \& Patton, 2016; X. Li, Karimi, Wang, Lu, \& Guo, 2018). Among FE techniques, there is an upward trend in the use of advanced observers (Gao et al., 2015). Sliding mode observers are used in (X. Wang, Tan, \& Zhou, 2017; Yin, Gao, Qiu, \& Kaynak, 2017), adaptive observers are applied in (X.-J. Li, Yan, \& Yang, 2018; Rodrigues, Hamdi, Theilliol, Mechmeche, \& BenHadj Braiek, 2015) and iterative observer schemes are studied in (Huang, Zhang, Guo, \& Wu, 2018). Augmented observers, which consider the faults as additional states, have received considerable attention (Gao, 2015; Wu, Feng, \& Duan, 2012; Gao \& Ho, 2004). Especially, proportional integral (PI) observers have been intensively studied (Chang, 2006; X. Li \& Zhu, 2015; Wu \& Duan, 2007) and applied (X. Liu, Gao, \& Zhang, 2018; Rotondo, Cristofaro, Johansen, Nejjari, \& Puig, 2016) in the last two decades.

One of the main problems in the use of common FE techniques for active FTC arises when the faults affecting a system are not isolable and it is not possible to build standard observers. Hence, most FE works conservatively consider that the faults verify isolability conditions, e.g., (X. Li et al., 2018; Witczak, Buciakowski, 
Puig, Rotondo, \& Nejjari, 2016). A solution to deal with non-isolable faults is the use of a bank of generalised observers (J. Chen \& Patton, 2012; Ding, 2008). However, these schemes are usually implemented from a FDI perspective and each observer in the bank is used to provide a residual signal which is sensitive to all but one fault, e.g. (Dong, Wu, \& Yang, 2017). Thus, there is a need to develop more FE approaches based on banks of observers for systems with non-isolable faults.

Another problem in the use of FE for active FTC schemes is the misleading effect produced when feedforwarding non-zero fault estimates in fault-free scenarios. The residual signal in FDI and the fault estimation signal in FE are subjected not only to faults but also to disturbances, which may deviate the estimation outputs. In the FDI framework, in order to make the residual signal sensitive to faults but robust against disturbances, structural methods such as the parametric eigenstructure assignment approach (Patton \& Chen, 2000) and the unknown input observer (UIO) approach (Hassanabadi, Shafiee, \& Puig, 2016; Ziyabari \& Shoorehdeli, 2017) are well-known. Alternatively, numerical approaches based on optimization methods that use the $\mathcal{H}_{\infty} / \mathcal{H}_{-}$and the $\mathcal{H}_{\infty} / \mathcal{H}_{\infty}$ indices have gained more attention in FDI research owing to its wide applicability (Y. Li, Karimi, Zhong, Ding, \& Liu, 2018; Ahmadizadeh, Zarei, \& Karimi, 2014; Aouaouda, Chadli, Shi, \& Karimi, 2015). In the FE framework, multiobjective optimization design approaches are also used in works as (Witczak et al., 2016; Rodrigues et al., 2015; Gao, Liu, \& Chen, 2016). In order to give further physical interpretation to the indices involved in the optimization problem, (P. Zhang \& Ding, 2008; W. Chen, Khan, Abid, \& Ding, 2011) propose to use the trade-off between the fault detection rate (FDR) and the false alarm rate (FAR). This trade-off is used in recent works as (Zhong, Zhang, Ding, \& Zhou, 2017; Zhao, Shen, \& Wang, 2017) and it is of practical importance in FDI applications. However, although the FAR and the FDR are suitable for FDI methods based on residuals, these indices give little information about other important issues in estimation-based methods such as the size of the faults which are susceptible to occur, the dynamic behaviour or the steady-state accuracy of the results. Some initial approaches considering a few of these issues can be found in works as (Peñarrocha, Dolz, \& Sanchis, 2015; Sales-Setién, Peñarrocha, Dolz, \& Sanchis, 2016; K. Zhang, Hao, Chen, Ding, \& Peng, 2015). The iterative design procedures of FDI residuals in (Peñarrocha et al., 2015) involve bounds of the minimum size of the diagnosable faults (MDF), bounds of the FAR and a decay ratio representing the fault tracking ability of the residuals. In (Sales-Setién et al., 2016) a non-iterative design procedure with the MDF, the FAR and the Cumulative Squared Error (CSE) of the residuals is proposed. For its part, the methods in (K. Zhang et al., 2015) include new indices as the expected detection delay (EDD) for FDI in statistical processes. In all, as stated in (K. Zhang, Jiang, \& Shi, 2012), more research on designs considering the performance of model-based FE is needed.

A well-known benchmark for FDI and FTC of wind turbines is developed in (Odgaard, Stoustrup, \& Kinnaert, 2013). The benchmark takes account of a wide variety of the multiple and diverse faults to which a wind turbine is prone and it sets some FDI performance requirements. A wide variety of solutions based on residuals have been presented for this wind turbine FDI problem, see (Odgaard \& Stoustrup, 2012). Data-based approaches are proposed in works such as (Pashazadeh, Salmasi, \& Araabi, 2018). Nonetheless, model-based approaches, such as the ones presented in (Sanchez, Escobet, Puig, \& Odgaard, 2015; W. Chen, Ding, et al., 2011), are more common. UIOs are presented in (Sanchez et al., 2015) and Kalman filters are developed in (W. Chen, Ding, et al., 2011). However, there are not solutions that provide a priori performance-based designs of the fault diagnosers to guarantee the requirements in the benchmark. Hence, the FDI performance is generally tested through an $a$ posteriori analysis or simulations. Regarding FTC, both passive and residual-based active FTC strategies have been applied to wind turbines in (Sloth, Esbensen, \& Stoustrup, 2011; Blesa, Rotondo, Puig, \& Nejjari, 2014). In (X. Liu, Gao, \& Chen, 2017; Lan, Patton, \& Zhu, 2016; Shi \& Patton, 2015) active FTC strategies based on FE are applied to wind turbines. However, all these works assume that only certain faults among all the possible ones may affect the turbines. The same assumption is considered in the FE solution presented in (Witczak, Rotondo, Puig, Nejjari, \& Pazera, 2017).

\subsection{Contributions}

In this work, FE is achieved by means of PI observers. The fault estimates are then evaluated in statistical-based decision mechanisms to achieve fault isolation (FI). The main contribution of this work is the development of 
novel estimation performance-based design strategies of PI observers. In analogy to the integrated design of residual generators and evaluators in (P. Zhang \& Ding, 2008; W. Chen, Khan, et al., 2011), we also present novel co-design strategies in the FI framework. We formulate the co-design of PI observers and statisticalbased decision mechanisms guaranteeing a priori isolation performance requirements. Compared to the relevant existing literature, the novelties of the proposed designs are the following.

- Designs with a priori performance requirements. In most cases, the performance of FE and FDI strategies is tested a posteriori (e.g., (Blesa, Jiménez, Rotondo, Nejjari, \& Puig, 2015; L. Chen, Patton, \& Goupil, 2012)). Hence, the satisfaction of estimation or isolation performance requirements entails iterative procedures. The designs proposed in this work guarantee a priori performance requirements and, thus, we avoid iterative design procedures.

- Designs with individual performance requirements. The designs proposed in this work deal with the performance of each single fault estimation/isolation channel. This increases the design flexibility compared with most existing FE and FI designs, where the performance is jointly fixed for all the fault estimation/isolation channels (e.g. (Witczak et al., 2016; Gao et al., 2016)).

- Designs with time-domain performance indices. In an aim to bridging the gap between theory and practice, we use new performance indices providing further practical and physical interpretation to the norm bounds which are commonly used in FDI and FE designs (Witczak et al., 2016; X. Li et al., 2018). The proposed observer designs for FE deal with the trade-off between the tracking delays w.r.t. different fault signals and the covariances of the fault estimates due to noises. The proposed co-designs for FI deal with the following isolation performance indices: the false isolation rates, the minimum isolable faults and the isolation times.

- Single-step numerical designs of observers guaranteeing unknown input and interfault decoupling. The well-known design of UIOs requires algebraic constraints to achieve unknown input (UI) decoupling (J. Chen $\&$ Patton, 2012). Then, the remaining design freedom is used in a numerical second-step design to achieve certain requirements over other performance criteria (X. Liu et al., 2018; X. Li \& Zhu, 2015; Witczak et al., 2016). In this work, we propose to use the concept of degree of UI decoupling in order to numerically achieve UI decoupling and other performance requirements in a single-step multiobjective optimization problem. We also introduce the concept of degree of interfault decoupling to deal with the transient fault estimation error which occurs due to the appearance of faults in other channels (e.g., the simulation results in (Rotondo, Nejjari, Puig, \& Blesa, 2015; Salahshoor, Mosallaei, \& Bayat, 2008)). The observer performance indices (covariance due to noises, fault tracking delays and UI and interfault decoupling) are altogether formulated via matrix inequalities in a single-step optimization problem.

We generalise these design strategies to a scheme based on a bank of PI observers and statistical-based decision mechanisms that allow achieving FI and FE in systems where isolability conditions of faults do not hold. Assuming the non-simultaneity of certain number of faults, we extend the concept of residual-based generalised observers (J. Chen \& Patton, 2012; Dong et al., 2017) from a FE perspective.

To show the goodness of the proposed approaches, we apply the strategies presented in this work to the wellknown benchmark for FDI and FTC of wind turbines (Odgaard et al., 2013). Unlike works as (Witczak et al., 2017; Lan et al., 2016), which just consider the occurrence of a reduced number of possible faults, we achieve the estimation of all the faults affecting the turbines. We also isolate these faults with a priori guaranteed isolation performance indices.

\subsection{Structure and Notation}

The outline of this work is as follows. First, we state the problem in Section 2, where we propose a FE and FI strategy based on PI observers and decision mechanisms. In Section 3, we present a FE performance-based design of the observer. In Section 4, we include a co-design of the observer and decision mechanism for guaranteeing certain trade-off between isolation performance indices. In Section 5, we extend the problem to the case in which FI becomes necessary for FE because fault isolability conditions are not verified and standard augmented 
observers are not applicable. Finally, Section 6 presents the results of applying the proposed FE and FI techniques to the wind turbine problem. Section 7 summarises the main conclusions.

Throughout the paper, $\mathbb{R}$ denotes the set of real numbers. Expected value, probability and absolute value are denoted by $\mathbb{E}\{\cdot\}, \mathbb{P}\{\cdot\}$ and $|\cdot|$. Let $A$ be some matrix and $a$ be some vector. $A_{i j}$ denotes the element in the $i$-th row and $j$-th column of $A$ and $a_{i}$ denotes the $i$-th element in $a . A \preceq 0$ means that $A$ is negative semidefinite and similar applies to $\succeq$. The rank of matrix $A$ is represented as $\operatorname{rank}\{A\}$ and its trace is given by $\operatorname{tr}\{A\}$. Let $x$ be a stochastic process. We write $\|x(k)\|_{2}^{2} \triangleq x(k)^{T} x(k)$ for the Euclidean norm of vector $x(k)$ and $\|x(k)\|_{\infty} \triangleq \max _{i}\left|x_{i}(k)\right|$ for its max norm. $\|x\|_{2}^{2} \triangleq \sum_{k=0}^{\infty}\|x(k)\|_{2}^{2}$ denotes the $l_{2}$ norm of process $x$, $\|x\|_{R M S}^{2} \triangleq \lim _{k \leftrightarrow \infty} \frac{1}{K} \sum_{k=0}^{K-1}\|x(k)\|_{2}^{2}$ denotes its RMS norm and $\|x\|_{\infty} \triangleq \max _{k} \max _{i}\left|x_{i}(k)\right|$ denotes its $l_{\infty}$ norm. $I_{n}$ is the identity matrix of size $n \times n$ or of appropriate size when the subindex is omitted; similar applies to $0_{n \times n}$.

\section{Problem Formulation}

Let us consider the linear time-invariant (LTI) discrete-time system defined by

$$
\begin{aligned}
x(k+1) & =A x(k)+B u(k)+E f(k)+G v(k)+D d(k), \\
y(k) & =C x(k)+F f(k)+H v(k),
\end{aligned}
$$

where $x \in \mathbb{R}^{n_{x}}, y \in \mathbb{R}^{n_{y}}$ and $u \in \mathbb{R}^{n_{u}}$ are the state, output and known input vector; $v \in \mathbb{R}^{n_{v}}$ is the process and sensor noise vector and $d \in \mathbb{R}^{n_{d}}$ is the UI vector. Vector $f \in \mathbb{R}^{n_{f}}$ includes all the process, actuator and sensor faults $f_{i}\left(i=1, \ldots, n_{f}\right)$ that affect the system (J. Chen \& Patton, 2012) ${ }^{1}$. We define $M_{i}$ as the selection matrix verifying $f_{i}=M_{i} f$ (i.e., $M_{i}=\left[\begin{array}{lll}0_{1 \times i-1} & 1 & 0_{1 \times n_{f}-i}\end{array}\right]$ ). The following assumptions on the system (1) are made.

Assumption 1. The pair $(A, C)$ is observable.

Remark 1. Augmented observers require the observability of the model (1) as detailed in works as (Gao, 2015; Chang, 2006). Hence, Assumption 1 is not restrictive from a FE perspective.

Assumption 2. The faults are detectable, isolable among them and isolable from the UIs.

Remark 2. The transfer matrix from a fault $f_{i}$ to the outputs is given by $G_{f_{i}}(z)=C(z I-A)^{-1} E_{i}+F_{i}$, where $E_{i}$ and $F_{i}$ represent the $i$-th column of $E$ and $F$, respectively. The transfer matrix from the UIs to the outputs

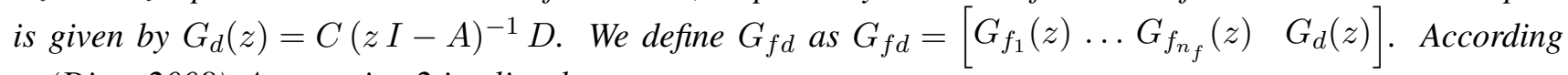
to (Ding, 2008), Assumption 2 implies that

$$
\begin{aligned}
G_{f_{i}}(z) & \neq 0, \quad \forall i \\
\operatorname{rank}\left\{G_{f d}(z)\right\} & =\sum_{i=1}^{n_{f}} \operatorname{rank}\left\{G_{f_{i}}(z)\right\}+\operatorname{rank}\left\{G_{d}(z)\right\} .
\end{aligned}
$$

The detectability of the faults in Assumption 2 is a necessary condition for FDI and FE (J. Chen \& Patton, 2012; Ding, 2008). The isolability condition in Assumption 2 is also standard in these frameworks. However, this condition becomes more restrictive as the number of UIs and faults increases (J. Chen \& Patton, 2012; Ding, 2008). Section 5 includes estimation and isolation strategies for the case in which the isolability condition in Assumption 2 does not hold.

Assumption 3. The noises are zero-mean and of known covariance, i.e., $\mathbb{E}\left\{v v^{T}\right\}=V$. The UIs are normbounded, i.e., $\|d\|_{\infty} \leq \bar{d}$.

\footnotetext{
${ }^{1}$ The proposed method entails a more general approach compared with some other existing works that only consider either actuator faults (Rodrigues et al., 2015) or sensor faults (Aouaouda et al., 2015; M. Liu \& Shi, 2013).
} 
Remark 3. Vector $v$ takes account of zero-mean stochastic disturbances while vector $d$ takes account of nonzero-mean norm-bounded disturbances. Other disturbances may be also considered in the model (1) by means of decomposing it into a component included in vector $v$ and a component included in vector $d$. Pure process noise $\left(v^{p}\right)$ and pure sensor noise $\left(v^{s}\right)$ can be modelled by means of zeroing the appropriate columns of $G$ and $H$ (i.e., $v=\left[\begin{array}{l}v^{p} \\ v^{s}\end{array}\right]$ with $G=\left[\begin{array}{ll}G^{p} & 0\end{array}\right]$ and $H=\left[\begin{array}{ll}0 & H^{s}\end{array}\right]$ ). For its part, the UI vector can be also used to describe a number of different kinds of norm-bounded modelling uncertainties (J. Chen, Patton, \& Zhang, 1996) (e.g., $d=\Delta A x$ with $\Delta A$ being the uncertainty regarding the state matrix $\left.{ }^{2}\right)$.

Assumption 4. The fault discrete derivative $\delta(k)=f(k+1)-f(k)$ is norm-bounded, i.e., $\|\delta\|_{\infty} \leq \bar{\delta}$.

Any fault signal verifying Assumption 4 (i.e., fault signals with norm-bounded fault discrete derivative $\delta$ ) can be modelled as

$$
\begin{aligned}
\xi(k+1) & =A_{F} \xi(k)+B_{F} \delta(k), \\
f(k) & =C_{F} \xi(k),
\end{aligned}
$$

with

$$
A_{F}=I_{n_{f}}, B_{F}=I_{n_{f}}, C_{F}=I_{n_{f}} .
$$

Remark 4. Assumption 4 is fairly general because it allows considering a wide range of fault signals which are common in practical applications. For instance, a step (or abrupt) fault signal is generated through (2) with an impulse signal $\delta$, and a ramp (or drift) fault signal is generated through (2) with a step signal $\delta$. Note that Assumption 4 does not imply any restriction over the upper bound of the fault vector $f$, which reduces the conservatism compared with some other existing works (Rodrigues et al., 2015; M. Liu \& Shi, 2013).

Remark 5. An augmented observer is based on an augmented model including both the system dynamics and the fault dynamics (Gao, 2015; Gao \& Ho, 2004). In the FE literature, fault dynamics verifying Assumption 4 are widely utilised leading to the so-called proportional integral (PI) observers (Chang, 2006; X. Liu et al., 2018), which are based on the fault model (2)-(3). A stable PI observer leads to bounded steady-state fault estimation errors when the system is subjected to fault signals verifying Assumption 4. For instance, it leads to zero steady-state errors under step faults and to constant steady-state errors under ramp faults.

Remark 6. Certain systems may be subjected to complex fault signal forms which do not verify Assumption 4. In such cases, the fault estimates provided by a PI observer would increase in value but the estimation errors would not be bounded. To ensure bounded fault estimation errors, the fault state-space matrices (3) must be replaced by more complex matrices. See, for instance, the state-space matrices in (Wu et al., 2012; Gao \& Ho, 2004) for fault signals in the more general form of a polynomial of the time leading to proportional multiple integral (PMI) observers. Hence, the model (3) and the strategies developed in this work are easily extensible to fault signals which do not verify Assumption 4.

The model (1) is thus augmented as

$$
\begin{aligned}
z(k+1) & =\mathcal{A} z(k)+\mathcal{B} u(k)+\mathcal{E} \delta(k)+\mathcal{G} v(k)+\mathcal{D} d(k), \\
y(k) & =\mathcal{C} z(k)+\mathcal{H} v(k), \\
f(k) & =\mathcal{R} z(k),
\end{aligned}
$$

where $z=\left[\begin{array}{l}x \\ \xi\end{array}\right]$ denotes the extended state vector. The state-space matrices in (4) verify $\mathcal{A}=\left[\begin{array}{cc}A & E C_{F} \\ 0 & A_{F}\end{array}\right]$, $\mathcal{B}=\left[\begin{array}{c}B \\ 0\end{array}\right], \mathcal{E}=\left[\begin{array}{c}0 \\ B_{F}\end{array}\right], \mathcal{G}=\left[\begin{array}{c}G \\ 0\end{array}\right], \mathcal{D}=\left[\begin{array}{ll}D^{T} & 0\end{array}\right]^{T}, \mathcal{C}=\left[\begin{array}{ll}C & F C_{F}\end{array}\right], \mathcal{H}=H$ and $\mathcal{R}=\left[\begin{array}{ll}0 & C_{F}\end{array}\right]$. The following

\footnotetext{
${ }^{2}$ In practice, FE is performed in stable (controlled or uncontrolled) systems. Hence, if $\Delta A$ is bounded, the uncertainty $d=\Delta A x$ is bounded as $x$ is also bounded.
} 
model-based PI observer is proposed to estimate the faults in (4)

$$
\begin{aligned}
\hat{z}(k+1) & =\mathcal{A} \hat{z}(k)+\mathcal{B} u(k)+L(y(k)-\mathcal{C} \hat{z}(k)), \\
\hat{f}(k) & =\mathcal{R} \hat{z}(k)+K(y(k)-\mathcal{C} \hat{z}(k)),
\end{aligned}
$$

where the design observer gain matrices $L$ and $K$ are of appropriate dimensions. Define the estimation errors $\tilde{z}(k)=z(k)-\hat{z}(k)$ and $\tilde{f}(k)=f(k)-\hat{f}(k)$. It follows that

$$
\begin{aligned}
\tilde{z}(k+1) & =\overline{\mathcal{A}} \tilde{z}(k)+\overline{\mathcal{G}} v(k)+\mathcal{D} d(k)+\mathcal{E} \delta(k), \\
\tilde{f}(k) & =\overline{\mathcal{R}} \tilde{z}(k)+\overline{\mathcal{H}} v(k),
\end{aligned}
$$

with $\overline{\mathcal{A}}=\mathcal{A}-L \mathcal{C}, \overline{\mathcal{G}}=\mathcal{G}-L \mathcal{H}, \overline{\mathcal{R}}=\mathcal{R}-K \mathcal{C}$ and $\overline{\mathcal{H}}=-K \mathcal{H}$. Applying the $\mathcal{Z}$ transform to (6), we get

$$
\tilde{f}(z)=\mathcal{G}_{\delta}(z) \delta(z)+\mathcal{G}_{d}(z) d(z)+\mathcal{G}_{v}(z) v(z),
$$

with $\mathcal{G}_{\delta}(z)=M(z) \mathcal{E}, \mathcal{G}_{d}(z)=M(z) \mathcal{D}, \mathcal{G}_{v}(z)=M(z) \overline{\mathcal{G}}+\overline{\mathcal{H}}$ and $M(z)=\overline{\mathcal{R}}(z I-\overline{\mathcal{A}})^{-1}$.

For isolation purposes, we set the following decision mechanisms $\left(i=1, \ldots, n_{f}\right)$ evaluating the fault estimates provided by (5):

$$
\left\{\begin{array}{ll}
\text { if }\left|\hat{f}_{i}(k)\right| \geq J_{i} & \text { Fault } i \\
\text { otherwise } & \text { No fault } i
\end{array},\right.
$$

where $J_{i}$ is the isolation threshold of the $i$-th fault and must be designed.

Remark 7. For FI, it is not necessary to evaluate the fault estimates $\hat{f}$ and appropriate simpler signals (i.e., isolation residuals) can be generated and evaluated instead, e.g., (J. Wang, Ge, Zhou, Wu, \& Jin, 2017; Hwang, Kim, Kim, \& Seah, 2010). In this work, the fault estimates are evaluated in isolation decision mechanisms in order to decide whether to feed or not an active FTC mechanism with these estimates.

In order to design the observer (5) and the decision mechanisms (8), one must choose the gain matrices $L$ and $K$ and the thresholds $J_{i}\left(i=1, \ldots, n_{f}\right)$. The main objective of this work is to solve the following problems

- To provide a design strategy of the observer for guaranteeing certain a priori estimation performance requirements.

- To provide a design strategy of the decision mechanisms for guaranteeing one a priori isolation performance requirement.

- To provide a co-design strategy of the observer and the decision mechanisms for guaranteeing more than one a priori isolation performance requirement.

\section{Fault Estimation}

\subsection{FE Performance Characterization}

The performance of the fault estimator (5) can be described using the following criteria:

e.1 the fault tracking speed,

e.2 the degree of interfault decoupling,

e.3 the degree of UI decoupling and

e.4 the noise attenuation. 
Each element $G_{\delta_{i j}}(z)$ of the transfer matrix $G_{\delta}(z)$ describes the fault tracking error that the $i$-th fault estimate (i.e., $\hat{f}_{i}$ ) experiences due to the variations of the fault in the channel $j$ (i.e., $\delta_{j}$ ). In particular, each diagonal element $G_{\delta_{i i}}(z)$ describes the error $\tilde{f}_{i}$ due to $\delta_{i}$ and each off-diagonal term $G_{\delta_{i j}}(z)$ with $j \neq i$ describes the coupling effect that occurs when $\tilde{f}_{i}$ increases due to the appearance of a fault in another channel $j \neq i$ (i.e., $\left.\delta_{j \neq i}\right)$. Since the fault tracking speed is strictly related to the fault tracking error, we make use of the $\mathcal{H}_{\infty}$ norm of the transfer functions $G_{\delta_{i i}}(z)\left(i=1, \ldots, n_{f}\right)$ to characterise the criterion e.1.. Likewise, the criterion e.2., which refers to the degree of decoupling between faults, can be characterised through the $\mathcal{H}_{\infty}$ norm of the transfer functions $G_{\delta_{i j}}(z)\left(i=1, \ldots, n_{f}, j=1, \ldots, i-1, i+1, \ldots, n_{f}\right)$.

Remark 8. There may also be a coupling effect when more than one fault vary simultaneously and it is also desirable to characterise this error. To cope with all these characterizations numerically, we bound each fault estimation error $\tilde{f}_{i}$ due to $\delta$ as

$$
\lim _{K \rightarrow \infty} \sum_{k=0}^{K} \tilde{f}_{i}(k)^{T} \tilde{f}_{i}(k) \leq \lim _{K \rightarrow \infty} \sum_{k=0}^{K} \delta(k)^{T} \Gamma^{i} \delta(k),
$$

where the term $\Gamma_{i i}^{i}$ stands for the $\mathcal{H}_{\infty}$ norm of $G_{\delta_{i i}}(z)$, the terms $\Gamma_{j j}^{i}(j \neq i)$ stand for the $\mathcal{H}_{\infty}$ norm of $G_{\delta_{i j}}(z)$ and the off-diagonal terms $\Gamma_{j l}^{i}(j \neq l \neq i)$ explain the behaviour of the $i$-th fault estimate towards simultaneous faults.

The dynamics in $\mathcal{G}_{d}(z)$ determine the effect of the UIs on the fault estimates. Thus, the criterion $\mathbf{e . 3}$ can be characterised through the $\mathcal{H}_{\infty}$ norm of $\mathcal{G}_{d}(z)$. Finally, we characterise the criterion e.4 through the covariance of the fault estimation error due to noises.

Remark 9. In the absence of UIs and faults, the presence of zero-mean noises leads to zero-mean fault estimation errors. In this case, the covariance of $\tilde{f}$, i.e., $\Sigma=\lim _{k \rightarrow \infty} \mathbb{E}\left\{\tilde{f}(k) \tilde{f}(k)^{T}\right\}$, is given by the Lyapunov equations

$$
\begin{aligned}
\Sigma^{z} & =\overline{\mathcal{A}} \Sigma^{z} \overline{\mathcal{A}}^{T}+\overline{\mathcal{G}} V \overline{\mathcal{G}}^{T}, \\
\Sigma & =\overline{\mathcal{R}} \Sigma^{z} \overline{\mathcal{R}}^{T}+\overline{\mathcal{H}} V \overline{\mathcal{H}}^{T},
\end{aligned}
$$

which we obtained from (7) with $\delta(z)=0, d(z)=0$ and using an internal realization of $G_{v}(z)$.

The requirements over these criteria can be thus translated into requirements over different $\mathcal{H}_{\infty}$ norms and covariance bounds of the fault estimation errors. In order to set multiobjective designs with different requirements over these criteria, we use the formulation based on matrix inequalities which is shown in Theorem 1.

Theorem 1. Consider the observer (5) applied to the system (4). If there exist any matrices $L$ and $K$, any positive scalar $\gamma_{d}$, any symmetric matrices $S, Q, \Xi, P_{i}$ and any diagonal matrices $\Gamma^{i}\left(i=1, \ldots, n_{f}\right)$ fulfilling

$$
\begin{aligned}
& {\left[\begin{array}{cccc}
Q & Q \overline{\mathcal{A}} & Q \mathcal{D} & 0 \\
\overline{\mathcal{A}}^{T} Q & Q & 0 & \overline{\mathcal{R}}^{T} \\
\overline{\mathcal{D}}^{T} Q & 0 & \gamma_{d} I & 0 \\
0 & \overline{\mathcal{R}} & 0 & I
\end{array}\right] \succeq 0,} \\
& {\left[\begin{array}{ccc}
S & S \overline{\mathcal{A}} & S \overline{\mathcal{G}} V \\
\overline{\mathcal{A}}^{T} S & S & 0 \\
V \overline{\mathcal{G}}^{T} S & 0 & V
\end{array}\right] \succeq 0,\left[\begin{array}{ccc}
\Xi & \overline{\mathcal{R}} & \overline{\mathcal{H}} V \\
\overline{\mathcal{R}}^{T} & S & 0 \\
V \overline{\mathcal{H}}^{T} & 0 & V
\end{array}\right] \succeq 0,} \\
& {\left[\begin{array}{cccc}
P_{i} & P_{i} \overline{\mathcal{A}} & P_{i} \mathcal{E} & 0 \\
\overline{\mathcal{A}}^{T} P_{i} & P_{i} & 0 & \overline{\mathcal{R}}^{T} M_{i}^{T} \\
\overline{\mathcal{E}}^{T} P_{i} & 0 & \Gamma^{i} & 0 \\
0 & M_{i} \overline{\mathcal{R}} & 0 & I
\end{array}\right] \succeq 0, \quad i=1, \ldots, n_{f} ;}
\end{aligned}
$$

the following statements hold: 
(i) In the absence of UIs, noises and faults, the extended state estimation error converges to zero.

(ii) The fault estimation error due to UIs is bounded $a s^{3}$

$$
\|\tilde{f}\|_{R M S}^{2} \leq \gamma_{d}\|d\|_{R M S}^{2} .
$$

(iii) The covariance of the fault estimation error due to noises is bounded as

$$
\Sigma \preceq \Xi .
$$

(iv) The fault estimation error due to fault variations is bounded as ${ }^{4}$

$$
\left\|\tilde{f}_{i}\right\|_{R M S}^{2} \leq \sum_{j=1}^{n_{f}} \Gamma_{j j}^{i}\left\|\delta_{j}\right\|_{R M S}^{2} .
$$

\section{Proof. See Appendix A}

Remark 10. Optimization-based FE strategies usually characterise the performance of the fault estimation error vector $\tilde{f}$ w.r.t. the UIs $d$, the noises $v$ and the fault variations $\delta$, e.g., (Gao et al., 2016; Witczak et al., 2016; Lan $\&$ Patton, 2017). In this work, we alternatively characterise the performance of each fault estimation error $\tilde{f}_{i}$ w.r.t. the noises $v$ (using the bound $\Xi_{i i}$ ) and w.r.t. each fault variation $\delta_{j}$ (using the bound $\Gamma_{j j}^{i}$ ). This approach allows designing estimators satisfying in each fault channel different trade-offs between fault sensitivity and noise attenuation, which is of practical interest in engineering applications. For its part, we just characterise the performance of the entire fault estimation error vector $\tilde{f}$ w.r.t. the UIs d because we desire to design PI observers ensuring UI decoupling.

\subsection{Observer Design with FE Performance Requirements}

Let us design the gain matrices $L$ and $K$ of the observer (5) for satisfying different requirements over the criteria e.1, e.2, e.3 and e.4.

From Theorem 1, we deduce that if $\Gamma_{i i}^{i}$ in (12) verifies

$$
\Gamma_{i i}^{i} \leq \bar{\Gamma}_{i i}^{i},
$$

the fault tracking error of the estimate $\hat{f}_{i}$ w.r.t. the variations described by $\delta_{i}$ is bounded by $\bar{\Gamma}_{i i}^{i}$. The fault estimation error can be bounded using the constraint (16); however, from a reliability perspective, constraints over the criterion e.1 may be of more practical interest. Since the fault tracking speed depends not only on the fault tracking error but also on the fault signal form, we can choose $\bar{\Gamma}_{i i}^{i}$ to ensure certain fault tracking speed w.r.t. an specific fault signal form verifying Assumption 4. Conservatively, we consider a ramp fault of slope $\Delta_{i} \neq 0$ occurring in the $i$-th fault channel ${ }^{5}$. In this case, the fault tracking speed may be described by the steady-state fault estimation delay $T_{i}$ which is bounded as $T_{i} \leq \sqrt{\Gamma_{i i}^{i}}$. To prove it, note that a ramp fault signal is generated through a constant signal $\delta_{i}$ (i.e., $\delta_{i}(k)=\Delta_{i}$ ) and the steady-state fault estimation error is also constant and equal to

$$
\lim _{k \rightarrow \infty} \tilde{f}_{i}(k)=\lim _{z \rightarrow 1}\left(1-z^{-1}\right) G_{\delta_{i i}}(z) \frac{\Delta_{i}}{1-z^{-1}}=G_{\delta_{i i}}(1) \Delta_{i}=\overline{\tilde{f}}_{i} .
$$

From the item (iv) in Theorem 1, we have that if $d=0$ and $v=0$,

$$
\frac{1}{K} \sum_{k=0}^{K} \tilde{f}_{i}(k)^{2} \leq \frac{1}{K} \sum_{k=0}^{K} \Gamma_{i i}^{i} \delta_{i}(k)^{2} .
$$

\footnotetext{
${ }^{3}$ It is also bounded as $\|\tilde{f}\|_{R M S}^{2} \leq \gamma_{d} n_{d}^{2} \bar{d}^{2}$ because $\|d\|_{R M S} \leq n_{d}\|d\|_{\infty}$ and $\|d\|_{\infty} \leq \bar{d}$.

${ }^{4}$ It is also bounded as $\left\|\tilde{f}_{i}\right\|_{R M S}^{2} \leq \sum_{j=1}^{n_{f}} \Gamma_{j j}^{i} \bar{\delta}^{2}$ because $\left\|\delta_{j}\right\|_{R M S} \leq\|\delta\|_{\infty}$ and $\|\delta\|_{\infty} \leq \bar{\delta}$.

${ }^{5} \mathrm{~A}$ constraint regarding the fault tracking speed w.r.t. ramp faults is conservative because it covers the worst-case fault signal form considered in Assumption 4 (i.e., a ramp fault of slope $\Delta_{i}=\bar{\delta}$ ).
} 
Provided that $\delta_{i}(k)=\Delta_{i}$, taking the limit when $K \rightarrow \infty$, and computing the square root of the result leads to $\left|\overline{\tilde{f}}_{i}\right| \leq \sqrt{\Gamma_{i i}^{i}} \Delta_{i}$. Taking into account that the slope $\Delta_{i}$ describes the proportionality between the increase of $f_{i}$ and the time elapsed between two different samples, we get the previous bound. Then, if $\Gamma_{i i}^{i}$ verifies (16), the estimation delay $T_{i}$ under ramp faults is bounded as $T_{i} \leq \sqrt{\bar{\Gamma}_{i i}^{i}}$.

Remark 11. Other fault signal forms verifying Assumption 4 could be considered to achieve a constraint over the criterion $e .1$. For instance, consider a constant fault of size $\bar{f}_{i} \neq 0$ occurring in the $i$-th fault channel. In this case, the fault tracking speed may be described by the cumulative squared error defined as $E_{i}=\left\|\tilde{f}_{i}(k)\right\|_{2}^{2}$. It is straightforward to prove that $E_{i} \leq \Gamma_{i i}^{i} \bar{f}_{i}^{2}$. Then, if $\Gamma_{i i}^{i}$ verifies (16), the cumulative squared error $E_{i}$ under unitary step faults is bounded as $E_{i} \leq \bar{\Gamma}_{i i}^{i}$.

Regarding the criterion $\mathbf{e . 2}$, if $\Gamma_{j j}^{i}$ in (12) verifies

$$
\Gamma_{j j}^{i} \leq \bar{\Gamma}_{j j}^{i},
$$

certain degree of interfault decoupling between $\hat{f}_{i}$ and $\delta_{j \neq i}$ is guaranteed. We define perfect interfault decoupling as the characteristic of an estimator verifying $\Gamma_{j j}^{i}=0$ for all $i$ and for all $j \neq i$. A numerically sound way of adding these constraints to a semidefinite programming problem involving (12) is to set the constraint (17) for all $i$ and for all $j \neq i$ and to fix

$$
\bar{\Gamma}_{j j}^{i}:=\epsilon_{j j}
$$

with $\epsilon_{j j}=\varepsilon \operatorname{tr}\{\Xi\} / \bar{f}_{j}, \varepsilon$ being a small number (e.g., $\varepsilon \leq 10^{-6}$ ) and $\bar{f}_{j}$ being the maximum expected value of the $j$-th fault, which can be derived from the physical constraints of the system. The value (18) in the constraint (17) makes the estimation error due to fault variations in other channels negligible w.r.t. the estimation error due to noises and we claim that practical interfault decoupling is achieved whenever (17)-(18) is satisfied for all $i$ and for all $j \neq i$. For its part, the use of diagonal matrices $\Gamma^{i}$ cancels the errors due to simultaneous variations of faults (i.e., the fault estimation error due to the product $\delta_{j} \delta_{l}$ with $j \neq l$ is cancelled).

Similarly, certain degree of decoupling from the UIs (criterion e.3) is guaranteed if $\gamma_{d}$ in (10) verifies

$$
\gamma_{d} \leq \bar{\gamma}_{d}
$$

We define perfect UI decoupling as the characteristic of an estimator verifying $\gamma_{d}=0$. Similarly to (18), we numerically address this issue through

$$
\bar{\gamma}_{d}:=\epsilon_{d}
$$

with $\epsilon=\varepsilon \operatorname{tr}\{\Xi\} / \bar{d}$. Thus, if (19)-(20) is fulfilled, the fault estimation error due to UIs becomes negligible w.r.t the error due to noises and we claim that practical UI decoupling is achieved.

Remark 12. Note that certain degree of interfault and UI decoupling is achievable regardless of the isolability of the faults and UIs. Perfect interfault and UI decoupling are achievable because the system (1) verifies Assumption 2 (i.e., the faults are isolable among them and from the UIs). If perfect UI decoupling is achieved, claims (iii) and (iv) in Theorem 1 do also hold in the presence of UIs. We consider that they also hold when practical UI decoupling is guaranteed and the UIs are present in the system.

Remark 13. The most extended strategy to build an observer guaranteeing perfect UI decoupling is the use of some algebraic constraints as the ones presented in (X. Liu et al., 2018; Gao et al., 2016; Ding, 2008) for the design of UIOs. Then, the remaining design freedom can be used in a second-step observer design to achieve certain requirements over other performance criteria. Alternatively, we propose to use the numerical constraints (19)-(20) in a design problem involving (10) to achieve practical UI decoupling. The proposal leads to an homogeneous formulation of all the performance criteria and allows achieving practical UI decoupling together with other estimation performance requirements in a single-step multiobjective optimization problem. Practical UI decoupling refers then to the numerical approach to achieve UI decoupling. In practice, numerical (or practical) UI decoupling is equivalent to structural (or perfect) UI decoupling, which is achieved using algebraic constraints. 
Table 1: Overview of observer design strategies for guaranteeing estimation performance requirements.

\begin{tabular}{llc}
\hline Target & \multicolumn{1}{c}{$\begin{array}{c}\text { Target } \\
\text { Formulation }\end{array}$} & $\begin{array}{c}\text { Observer } \\
\text { Design }\end{array}$ \\
\hline Practical UI decoupling & $\gamma_{d} \leq \epsilon_{d}$ & Optimization \\
Practical interfault decoupling & $\Gamma_{j j}^{i} \leq \epsilon_{j j}, \forall i, j \neq i$ & problem \\
Bounded ramp fault estimation delays & $T_{i} \leq T_{i}^{*}, \forall i$ & (22)-(23) \\
Minimised marginal variances due to noises & $\min f\left(\Sigma_{11}, \ldots, \Sigma_{n_{f} n_{f}}\right)$ & \\
\hline Practical UI decoupling & $\gamma_{d} \leq \epsilon_{d}$ & Optimization \\
Practical interfault decoupling & $\Gamma_{j j}^{i} \leq \epsilon_{j j}, \forall i, j \neq i$ & problem \\
Bounded marginal variances due to noises & $\Sigma_{i i} \leq \Sigma_{i i}^{*}, \forall i$ & $(24)-(25)$ \\
Minimised ramp fault estimation delays & $\min f\left(T_{1}, \ldots, T_{n_{f}}\right)$ & \\
\hline
\end{tabular}

Finally, if matrix $\Xi$ in (11) verifies

$$
\Xi_{i i} \leq \bar{\Xi}_{i i}
$$

certain noise attenuation (criterion e.4) is guaranteed in the $i$-th fault estimation channel. Particularly, (21) implies that the marginal variance of $\tilde{f}_{i}$ is bounded as $\Sigma_{i i} \leq \bar{\Xi}_{i i}$.

The following two multiobjective design strategies, summarised in Table 1, show a proposal of how to use these results for designing the fault estimator (5) guaranteeing different estimation performance requirements.

Strategy 1. Let us assume that we want to design a fault estimator (5) that minimises certain linear function $f(\cdot)$ of the marginal variances of the fault estimation errors due to noises while it guarantees practical UI and interfault decoupling, and certain fault estimation delays under ramp faults, with $T_{i}^{*}$ being the maximum allowed delay in the $i$-th fault channel. To address this design, we solve the following optimization problem

$$
\begin{array}{ll}
\text { minimise } & f\left(\Xi_{11}, \ldots, \Xi_{n_{f} n_{f}}\right) \\
\text { subject to } & \{(10)-(12),(16)-(20), \forall i, j \neq i\}
\end{array}
$$

with

$$
\bar{\Gamma}_{i i}^{i}:=T_{i}^{*}
$$

in (16) and along the variables $S, Q, P^{i}, \Xi, \Gamma^{i}, K, L$ and $\gamma_{d}$ with $i=1, \ldots, n_{f}$ and $j=1, \ldots, n_{f}$.

Strategy 2. Let us assume that we want to design a fault estimator (5) that minimises certain linear function $f(\cdot)$ of the fault estimation delays under ramp faults while it guarantees practical UI and interfault decoupling, and certain marginal variance of each fault estimation error, with $\Sigma_{i i}^{*}$ being the variance requirement in the $i$-th fault channel. To address this design, we solve the following optimization problem

$$
\begin{array}{ll}
\text { minimise } & f\left(\Gamma_{11}^{1}, \ldots, \Gamma_{n_{f}}^{n_{f}}\right) \\
\text { subject to } & \{(10)-(12),(17)-(21), \forall i, j \neq i\}
\end{array}
$$

with

$$
\bar{\Xi}_{i i}:=\Sigma_{i i}^{*}
$$

in (21) and along the variables $S, Q, P^{i}, \Xi, \Gamma^{i}, K, L$ and $\gamma_{d}$ with $i=1, \ldots, n_{f}$ and $j=1, \ldots, n_{f}$.

\subsubsection{Conservativeness and Solvability of the Observer Design}

The multiobjective optimization problems in Strategy 1 and Strategy 2 are based on the results of Theorem 1, whose conditions are standard in norm-based designs (Guerra, Márquez, Kruszewski, \& Bernal, 2018; Zhou, Doyle, Glover, et al., 1996). The use of independent closed-loop Lyapunov functions $Q$ and $P_{i}\left(i=1, \ldots, n_{f}\right)$, 
which are different from the matrix bound $S$, guarantee non-conservative designs based on the inequalities (10)(12) because the estimation error model (6) is LTI. However, these designs become nonlinear optimization problems entailing bilinear matrix inequalities (BMIs).

These nonlinear problems can be solved using different solvers such as the ones presented in (Henrion, Löfberg, Kočvara, \& Stingl, 2005; Kočvara \& Stingl, 2003). Unfortunately, these solvers introduce certain degree of conservatism because they only guarantee local solutions. Alternatively, it is possible to iteratively solve the BMIs through a sequence of problems of linear matrix inequalities (LMIs) following different approaches such as the ones presented in (El Ghaoui, Oustry, \& AitRami, 1997). Note that recovering convexity by enforcing $Q=S=P_{i}$ for all $i$ is not suitable because this approach is too conservative. However, a compromise solution can be also adopted by introducing a slack variable as detailed in works as (Guerra et al., 2018; de Oliveira, Bernussou, \& Geromel, 1999).

Practical interfault and UI decoupling, which are required in Strategy 1 and Strategy 2, are achievable because the system (1) verifies Assumption 2. Hence, the solvability of the optimization problems (22) and (24) depends on the restrictiveness of the values which are chosen for the performance requirements $T_{i}^{*}$ and $\Sigma_{i i}^{*}$ $\left(i=1, \ldots, n_{f}\right)$, respectively. The solvability limits of the performance requirements can be found using the following problems.

- The most restrictive requirements $T_{i}^{*}\left(i=1, \ldots, n_{f}\right)$ guaranteeing the solvability of the design problem in Strategy 1 satisfy $T_{i}^{*}:=\Gamma_{i i}^{i}$, with $\Gamma_{i i}^{i}$ being the solution to the following problem:

$$
\begin{array}{ll}
\operatorname{minimise} & f\left(\Gamma_{11}^{1}, \ldots, \Gamma_{n_{f} n_{f}}^{n_{f}}\right) \\
\text { subject to } & \{(10),(12),(16)-(20), \forall i, j \neq i\}
\end{array}
$$

along the variables $S, Q, P^{i}, \Gamma^{i}, K, L$ and $\gamma_{d}$ with $i=1, \ldots, n_{f}$ and $j=1, \ldots, n_{f}$.

- The most restrictive requirements $\Sigma_{i i}^{*}\left(i=1, \ldots, n_{f}\right)$ guaranteeing the solvability of the design problem in Strategy 2 satisfy $\Sigma_{i i}^{*}:=\Xi_{i i}$, with $\Xi_{i i}$ being the solution to the following problem:

$$
\begin{array}{ll}
\operatorname{minimise} & f\left(\Xi_{11}, \ldots, \Xi_{n_{f} n_{f}}\right) \\
\text { subject to } & \{(10),(11),(17)-(21), \forall i, j \neq i\}
\end{array}
$$

along the variables $S, Q, P^{i}, \Xi, K, L$ and $\gamma_{d}$ with $i=1, \ldots, n_{f}$ and $j=1, \ldots, n_{f}$.

\section{Fault Isolation}

\subsection{FI Performance Characterization}

Motivated by the characterization presented in (J. Chen \& Patton, 2012; Ding, 2008), we describe the performance of the fault isolator (8) through the following indices:

i.1. the false isolation rates,

i.2. the minimum isolable faults,

i.3. the acknowledgement times and

i.4. the isolation times.

Let us first define a persistent fault $f_{i}$ satisfying

$$
\left\{\begin{array}{ll}
\left|f_{i}(k)\right|=0 & \text { if } k<k_{0} \\
\left|f_{i}(k)\right| \in\left(0, f_{i}\right) & \text { if } k \in\left[k_{0}, \underline{k}\right) \\
\left|f_{i}(k)\right| \geq f_{\underline{i}} & \text { if } k \geq \underline{k}
\end{array} .\right.
$$


We define the false isolation rate of the fault $i$, which we denote as $\phi_{i}$, as the probability of rising an isolation alarm of the fault $i$ when $f_{i}=0$ :

$$
\phi_{i}=\mathbb{P}\left\{\exists k:\left|\hat{f}_{i}(k)\right| \geq J_{i}\right\} .
$$

Provided $f_{j \neq i}=0, v=0$ and $d=0$, we define the minimum isolable fault $i$, which we denote as $\psi_{i}$, as the smallest value $f_{\underline{i}}$ that ensures the isolation of the fault (26):

$$
\psi_{i}=\left\{\begin{array}{l}
\min \underline{f}_{i} \\
\text { s.t. } \quad \exists k \geq \underline{k}:\left|\hat{f}_{i}(k)\right| \geq J_{i}
\end{array}\right\} .
$$

Under these conditions (i.e., $f_{j \neq i}=0, v=0$ and $d=0$ ), we define the acknowledgement time of the fault $i$, which we denote as $\vartheta_{i}$, as the time elapsed between $\underline{k}$ and the first sample of isolation of the fault (26):

$$
\vartheta_{i}=\left\{\begin{array}{l}
\min _{k \geq \underline{k}} k-\underline{k} \\
\text { s.t. }\left|\hat{f}_{i}(k)\right|>J_{i}
\end{array}\right\} .
$$

We define the isolation time of the fault $i$, which we denote as $\tau_{i}$, as the time elapsed between the appearance of the fault (26) at $k_{0}$ and the first sample of isolation of this fault:

$$
\tau_{i}=\underline{k}-k_{0}+\vartheta_{i}
$$

\subsection{Mechanisms Design with FI Requirements}

Assume that the model-based observer (5) has been designed through the strategies presented in Section 3.2 and the fault estimate $\hat{f}_{i}$ provided by such observer (with prefixed stabilising gains $L$ and $K$ ) is used in the decision mechanism (8). In the following, we show how to design the threshold $J_{i}$ of the decision mechanism (8) for guaranteeing certain requirement over one isolation performance index.

Regarding the index i.1, if $f_{i}=0$ and perfect UI and interfault decoupling are achieved, the fault estimate $\hat{f}_{i}$ is zero-mean and its variance is given by the marginal variance $\Sigma_{i i}$, which can be computed through (9). Then, through Chebyshev's inequality ${ }^{6}$ and the definition (27), we have that

$$
\phi_{i} \leq \Sigma_{i i} / J_{i}^{2},
$$

and we can set $J_{i}$ as

$$
J_{i}:=\sqrt{\Sigma_{i i} / \phi_{i}^{*}} .
$$

to guarantee the bound $\phi_{i} \leq \phi_{i}^{*}$.

Remark 14. Provided UI and interfault decoupling, if the noises $v$ are Gaussian, we have that $\hat{f}_{i} \sim \mathcal{N}\left(0, \Sigma_{i i}\right)$ and we can set $J_{i}$ to fix the index $i .1$ to $\phi_{i}^{*}$ as

$$
J_{i}:=\Phi_{Z}^{-1}\left(1-\phi_{i}^{*} / 2\right) \sqrt{\Sigma_{i i}},
$$

with $\Phi_{Z}^{-1}(\cdot)$ being the inverse cumulative distribution function of a standard normal variable ${ }^{7}$.

The minimum isolable fault $i$ depends on the form of the fault signal $f_{i}$, see the definition (28). Then, we can just ensure certain index i.2 w.r.t. an specific fault signal form verifying Assumption 4 and the conditions (26). The straightforward case is the occurrence of a non-zero step fault in the $i$-th fault channel because the minimum isolable fault coincides with the threshold of the isolation mechanism, i.e.,

$$
\psi_{i} \equiv J_{i}
$$

Hence, we can fix the minimum isolable constant fault $i$ to $\psi_{i}^{*}$ by setting

$$
J_{i}:=\psi_{i}^{*}
$$




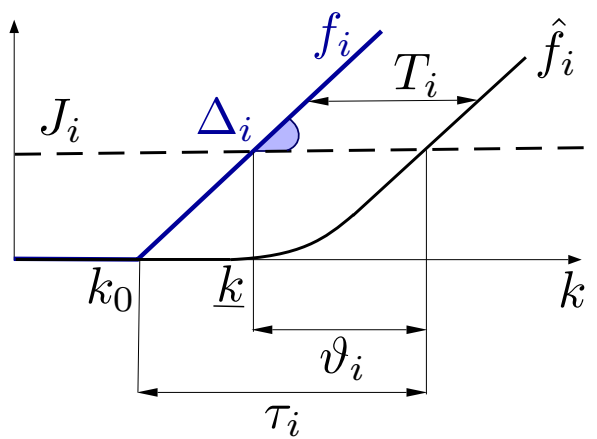

Figure 1: Time isolation indices in the presence of a ramp fault.

Table 2: Overview of mechanism design strategies for guaranteeing one isolation performance requirement.

\begin{tabular}{lcc}
\hline Target & $\begin{array}{c}\text { Target } \\
\text { Formulation }\end{array}$ & $\begin{array}{c}\text { Mechanism } \\
\text { Design }\end{array}$ \\
\hline Bounded false isolation rate & $\phi_{i} \leq \phi_{i}^{*}$ & Obtain $\Sigma_{i i}$ with (9) Equality (32) \\
\hline Certain minimum isolable constant fault & $\psi_{i} \equiv \psi_{i}^{*}$ & Equality (35) \\
\hline Certain ramp fault isolation time (slope $\left.\Delta_{i}\right)$ & $\tau_{i} \equiv \tau_{i}^{*}$ & Equality (37) \\
\hline & \multicolumn{1}{|}{${ }^{\dagger}$ Equality (33) with Gaussian noises ensures $\phi_{i} \equiv \phi_{i}^{*}}$.
\end{tabular}

The time indices i.3 and i.4 do also depend on the form of the exogenous fault signal $f_{i}$, see (29) and (30). Then, we can just ensure certain time indices w.r.t. an specific fault signal form verifying Assumption 4 and the conditions (26). In analogy to the estimation performance criterion e.1, we consider the occurrence of a ramp fault of slope $\Delta_{i} \neq 0$ in the $i$-th fault channel, see Fig.1. If the estimation error has achieved the steady state when the fault exceeds $J_{i}$, the acknowledgement time of the fault $i$ is the steady-state estimation delay $T_{i}$ (i.e., $\vartheta_{i} \equiv T_{i}$ ) and the isolation time of the fault $i$ satisfies

$$
\tau_{i} \equiv J_{i} / \Delta_{i}+T_{i}
$$

where $J_{i} / \Delta_{i}$ characterises the time that the ramp fault requires to achieve $J_{i}$. In all, $\vartheta_{i} \equiv T_{i}$ is determined by the observer and it cannot be modified by varying $J_{i}$. Provided certain $T_{i}$, we can set

$$
J_{i}:=\Delta_{i}\left(\tau_{i}^{*}-T_{i}\right)
$$

to fix the isolation time to $\tau_{i}^{*}$ (i.e., $\tau_{i} \equiv \tau_{i}^{*}$ ) if $\tau_{i}^{*}>T_{i}$ and $\Delta_{i}$ is known. Note that the slope $\Delta_{i}$ is not generally known and requirements over the acknowledgement time $\vartheta_{i}$ are more general.

The mechanism design strategies presented in this section are summarised in Table 2. Note that once $J_{i}$ is designed to guarantee one isolation performance index, the other indices can be computed through (31), (34) and (36).

\subsection{Co-design with FI Requirements}

The strategies presented in Section 4.2 show how to design the decision mechanism (8) to ensure one requirement over the index i.1, i.2. or i.4 when the gain matrices $L$ and $K$ of the observer (5) are prefixed (i.e., the gains are already designed). In order to achieve an isolator guaranteeing two or more requirements over these indices, it is necessary to perform a co-design of the observer (5) and the decision mechanisms (8). The following

\footnotetext{
${ }^{6}$ If $a>0$ and $x$ is a random variable of mean $\mu$ and variance $\sigma$, then $\mathbb{P}\{|x-\mu|>a \sigma\} \leq 1 / a^{2}$.

${ }^{7}$ Note that the threshold $J_{i}$ defined as (33) ensures $\phi_{i} \equiv \phi_{i}^{*}$ in the case of Gaussian noises while the threshold $J_{i}$ defined as (32) ensures the bound $\phi_{i} \leq \phi_{i}^{*}$ regardless of the statical distribution of the noises.
} 
strategies, summarised in Table 3, show a proposal of how to perform this co-design for guaranteeing more than one isolation performance requirement.

Strategy 3. Assume that we desire to ensure altogether certain false isolation rates $\phi_{i}^{*}\left(i=1, \ldots, n_{f}\right)$, certain minimum isolable constant faults $\psi_{i}^{*}\left(i=1, \ldots, n_{f}\right)$ and minimum acknowledgement times under ramp faults (and thus minimum isolation times under ramp faults). To ensure these requirements, we first design the observer (5) through Strategy 2 with the value

$$
\Sigma_{i i}^{*}:=\phi_{i}^{*} \psi_{i}^{* 2}
$$

in (25) for all $i$. Second, with the obtained gains $L$ and $K$, we compute $\Sigma$ through $(9)^{8}$ and we set the isolation thresholds through (32) with $\phi_{i}^{*}$ for all $i$.

Remark 15. If the noises $v$ that affect the system (1) are Gaussian, the constraint (38) can be replaced by

$$
\Sigma_{i i}^{*}:=\psi_{i}^{* 2} / \Phi_{Z}^{-1}\left(1-\phi_{i}^{*} / 2\right)^{2}
$$

and each isolation threshold can be set through (33) with $\phi_{i}^{*}$.

Strategy 4. Now, assume that we desire to ensure altogether certain false isolation rates $\phi_{i}^{*}\left(i=1, \ldots, n_{f}\right)$, certain acknowledgment times under ramp faults $\vartheta_{i}^{*}\left(i=1, \ldots, n_{f}\right)$ and we desire to minimise the minimum isolable faults. To ensure these requirements, we first design the observer (5) through Strategy 1 with the value

$$
T_{i}^{*}:=\vartheta_{i}^{*}
$$

in (23) for all $i$. Second, with the obtained gains $L$ and $K$, we compute $\Sigma$ through $(9)^{8}$ and we set the isolation thresholds through (32) (or (33) if the noises are Gaussian) with $\phi_{i}^{*}$ for all $i$.

Remark 16. Note that if we desire to ensure altogether certain acknowledgement times under ramp faults $\vartheta_{i}^{*}$ $\left(i=1, \ldots, n_{f}\right)$, certain minimum isolable constant faults $\psi_{i}^{*}\left(i=1, \ldots, n_{f}\right)$ and we desire to minimise the false isolation rates, we just have to design the observer (5) through Strategy 1 with the value $T_{i}^{*}:=\vartheta_{i}^{*}$ and set $J_{i}:=\psi_{i}^{*}$ for all $i$.

Remark 17. In order to achieve an isolator which guarantees certain isolation times $\tau_{i}^{*}$ under ramp faults of slope $\Delta_{i}\left(i=1, \ldots, n_{f}\right)$, certain false isolation rates $\phi_{i}^{*}\left(i=1, \ldots, n_{f}\right)$ while it minimises the minimum isolable constant faults, we must design the observer (5) through Strategy 1 with $T_{i}^{*}:=\tau_{i}^{*}-1 / \Delta_{i} \sqrt{\Xi_{i i} / \phi_{i}^{*}}$ in (23) for all $i$. The constraint (23) becomes, then, nonlinear. Provided this nonlinearity and given that slope $\Delta_{i}$ is generally unknown, we use Strategy 4 whenever requirements over time isolation indices appear in the co-design.

\section{$5 \quad$ FI and FE with a Bank of Observers}

In this section, we address the case in which the faults and the UIs in the system (1) are not isolable (i.e. $\left.\operatorname{rank}\left\{G_{f d}(z)\right\}<\sum_{i=1}^{n_{f}} \operatorname{rank}\left\{G_{f_{i}}(z)\right\}+\operatorname{rank}\left\{G_{d}(z)\right\}\right)$ and, thus, it is not possible to build model-based observers that guarantee both decoupling from the UIs and appropriate fault estimates. Then, we design several observers (i.e., a bank of observers), each of them taking only into account a subset of all the faults to which the system is prone. Assuming that all the faults in the system are not simultaneous, we build a bank of decision mechanisms for the bank of observers which enhances, first, FI and, then, FE.

Remark 18. As detailed in Remark 12, certain degree of UI decoupling is achievable regardless of the isolability of the faults from the UIs. In this section, we assume that practical UI decoupling is required and intermediate solutions guaranteeing certain degree of UI decoupling do not fulfil the required performance.

\footnotetext{
${ }^{8}$ Strategy 2 guarantees practical UI and interfault decoupling and thus, in the fault-free scenarios, signal $\hat{f}_{i}$ is zero-mean and its variance is given by the marginal variance $\Sigma_{i i}$.
} 
Table 3: Overview of co-design strategies for guaranteeing isolation performance requirements.

\begin{tabular}{|c|c|c|c|}
\hline Target & $\begin{array}{c}\text { Target } \\
\text { Formulation }\end{array}$ & $\begin{array}{c}\text { Observer } \\
\text { Design }\end{array}$ & $\begin{array}{l}\text { Mechanisms } \\
\text { Design }\end{array}$ \\
\hline $\begin{array}{l}\text { Bounded false isolation rates } \\
\text { Bounded minimum isolable constant } \\
\text { faults } \\
\text { Minimised ramp fault isolation times } \\
\text { Minimised ramp fault acknowledge- } \\
\text { ment times }\end{array}$ & $\begin{array}{l}\phi_{i} \leq \phi_{i}^{*}, \forall i \\
\psi_{i} \leq \psi_{i}^{*}, \forall i \\
\min f\left(\tau_{1}, \ldots, \tau_{n_{f}}\right) \\
\min f\left(\vartheta_{1}, \ldots, \vartheta_{n_{f}}\right)\end{array}$ & $\begin{array}{l}\text { Optimization problem } \\
\qquad \begin{array}{c}(24)-(25)^{\dagger} \\
\Sigma_{i i}^{*}:=\phi_{i}^{*} \psi_{i}^{* 2}, \forall i\end{array}\end{array}$ & $\begin{array}{c}\text { Obtain } \Sigma(9) \\
\text { Equality }(32)^{\ddagger}\end{array}$ \\
\hline $\begin{array}{l}\text { Bounded false isolation rates } \\
\text { Bounded ramp fault acknowledgement } \\
\text { times } \\
\text { Minimised minimum isolable constant } \\
\text { faults }\end{array}$ & $\begin{array}{l}\phi_{i} \leq \phi_{i}^{*}, \forall i \\
\vartheta_{i} \leq \vartheta_{i}^{*}, \forall i \\
\min f\left(\psi_{1}, \ldots, \psi_{n_{f}}\right)\end{array}$ & $\begin{array}{c}\text { Optimization } \\
\text { problem (24)-(25) } \\
T_{i}^{*}:=\vartheta_{i}^{*}, \forall i\end{array}$ & $\begin{array}{c}\text { Obtain } \Sigma(9) \\
\text { Equality }(32)^{\ddagger}\end{array}$ \\
\hline $\begin{array}{l}\text { Bounded minimum isolable constant } \\
\text { faults } \\
\text { Bounded ramp fault acknowledgement } \\
\text { times } \\
\text { Minimised false isolation rates }\end{array}$ & $\begin{array}{l}\psi_{i} \leq \psi_{i}^{*}, \forall i \\
\vartheta_{i} \leq \vartheta_{i}^{*}, \forall i \\
\min f\left(\phi_{1}, \ldots, \phi_{n_{f}}\right)\end{array}$ & $\begin{array}{c}\text { Optimization } \\
\text { problem (24)-(25) } \\
T_{i}^{*}:=\vartheta_{i}^{*}, \forall i\end{array}$ & Equality (35) \\
\hline
\end{tabular}

Remark 19. Consider the case in which certain requirements over estimation or isolation performance indices compromise FE or FI w.r.t. other performance indices. Although not being necessary in terms of isolability conditions, the use of a bank of observers leads to a better performance w.r.t the compromised indices at the cost of new restrictions over the simultaneity of faults. This situation gives a further motivation to the strategies developed in this section.

\subsection{Bank of Observers and Decision Mechanisms for FI and FE}

Let us denote the set of all possible faults as $S=\left\{f_{1}, \ldots, f_{n_{f}}\right\}$ and the set of the corresponding ordered indices as $\pi=\left\{1, \ldots, n_{f}\right\}$ (i.e., $\pi_{i}=i$ ). We split the model (1) into a bank of $m$ submodels. Each submodel $b$ (with $b=1, \ldots, m$ ) takes account of a subset $S^{b} \subset S$ of $n_{s}<n_{f}$ faults (with ordered indices $\pi^{b} \subset \pi$ ) while it ignores the other faults. Every fault of the system is at least considered by one submodel in the bank (i.e., $S=\bigcup_{b} S^{b}$ ) and $S^{b} \neq S^{c}$ for $b \neq c$. The number of submodels in the bank is thus

$$
m=\mathbf{C}_{n_{s}}^{n_{f}}=\frac{n_{f} !}{n_{s} !\left(n_{f}-n_{s}\right) !} .
$$

We denote the vector that stacks the faults which are taken into account by the $b$-th submodel as $f^{b}$ and the vector that stacks the faults which are ignored by this submodel as $f^{\backslash b}$. The $b$-th submodel is

$$
\begin{aligned}
x(k+1) & =A x(k)+B u(k)+E^{b} f^{b}(k)+G v(k)+D d(k), \\
y(k) & =C x(k)+F^{b} f^{b}(k)+H v(k),
\end{aligned}
$$

with $E^{b}$ and $F^{b}$ being the result of stacking the columns of $E$ and $F$ indexed by $\pi^{b}$. The size $n_{s}$ of the subsets $S^{b}$ must be chosen in order to guarantee the isolability of all the fault vectors $f^{b}$ in the presence of UIs. For all $b$, we must have that

$$
\operatorname{rank}\left\{G_{f^{b} d}(z)\right\}=\sum_{l=1}^{n_{s}} \operatorname{rank}\left\{G_{f_{l}^{b}}(z)\right\}+\operatorname{rank}\left\{G_{d}(z)\right\},
$$


with $G_{f_{l}^{b}}(z)=C(z I-A)^{-1} E_{l}^{b}+F_{l}^{b}$ and $G_{f^{b} d}(z)=\left[G_{f_{1}^{b}}(z) \ldots G_{f_{n_{s}}^{b}}(z) G_{d}(z)\right]$. Due to the additive nature of the faults and the UIs in (1), it is not possible to guarantee the condition (43) if $n_{s}>n_{y}-n_{d}$. Then, we set $n_{s}$ as the maximum number less than or equal to $n_{y}-n_{d}$ such that the condition (43) holds for all the submodels in the bank.

In analogy to (4), we augment each submodel (42) with the dynamics of the fault vector $f^{b}$ (i.e., $A_{F}^{b}=$ $I_{n_{s}}, B_{F}^{b}=I_{n_{s}}, C_{F}^{b}=I_{n_{s}}$ ). Then, likewise to (5), we build a bank of observers in the form of

$$
\begin{aligned}
\hat{z}^{b}(k+1) & =\mathcal{A}^{b} \hat{z}^{b}(k)+\mathcal{B}^{b} u(k)+L^{b}\left(y(k)-\mathcal{C}^{b} \hat{z}^{b}(k)\right), \\
\hat{f}^{b}(k) & =\mathcal{R}^{b} \hat{z}^{b}(k)+K^{b}\left(y(k)-\mathcal{C}^{b} \hat{z}^{b}(k)\right)
\end{aligned}
$$

with $L^{b}$ and $K^{b}$ being the observer gain matrices of the $b$-th observer of appropriate dimensions. Vector $\hat{f}^{b}$ is the estimated fault and $\hat{z}^{b}$ is the estimated extended state.

Note that (42) models the behaviour of the system (1) when the faults $f^{\backslash b}$ are not present in the system (i.e., $f^{\backslash b}=0$ ). Then, $\hat{f}^{b}$ is only reliable when $f^{\backslash b}=0$. We know that a fault $f_{i}$ is zero if $\hat{f}_{l}^{b}=0$ with $\pi_{l}^{b}=i$ for some estimator $b$. If $n_{s}$ or more simultaneous faults occur, there are no zero-value fault estimates and all the estimates provided by the bank are thus corrupted. This means that it is only possible to discern reliable estimates when no more than $n_{s}-1$ simultaneous faults are present in the system and that FE and FI are only possible if $n_{s}>1$. In all, Algorithm 1 summarises the strategy to build the bank of observers guaranteeing the isolation and estimation of the maximum possible number of simultaneous faults.

Remark 20. Assume that the condition (43) does not hold for all submodels $b$ if $n_{s}>1$.

- If (43) holds at least for some submodels $b$ when $n_{s}>1$ and all the faults $f_{i}\left(i=1, \ldots, n_{f}\right)$ are considered within these submodels, we extend them with $A_{F}^{b}=I_{n_{s}}, B_{F}^{b}=I_{n_{s}}, C_{F}^{b}=I_{n_{s}}$ and we build the corresponding observers (44). We extend the other submodels which do not verify (43) with $A_{F}^{b}=0_{n_{s} \times n_{s}}, B_{F}^{b}=I_{n_{s}}, C_{F}^{b}=I_{n_{s}}$ and we build the corresponding observers (44). In this case, the latter observers are only used for FI purposes and allow discerning the reliability of the outputs provided by the first group of observers, which are used for FE purposes. See the details in (Sales-Setien \& Peñarrocha-Alós, 2018)9.

- Otherwise, a transformation of the system, as proposed in (Z. Li, Mazars, Zhang, \& Jaimoukha, 2012), must be done (leading to new a fault vector $f$ ).

In noisy environments, there are not zero-value fault estimates. This means that decision mechanisms based on thresholds are necessary for both FI and FE. Likewise to (8), we set the following decision mechanism which enables FI when no more than $n_{s}-1$ simultaneous faults occur

$$
\left\{\begin{array}{ll}
\text { if }\left|\hat{f}_{l}^{b}(k)\right| \geq J_{l}^{b} \quad \forall(b, l): \pi_{l}^{b}=i & \text { Fault } i \\
\text { otherwise } & \text { No fault } i
\end{array} .\right.
$$

with $J_{l}^{b}$ being the isolation threshold of the $l$-th fault in the $b$-th bank.

For FE, we rely on $\hat{f}_{l}^{b}$ as an estimate of $f_{\pi_{l}^{b}}$ whilst no fault in $f^{\backslash b}$ has been isolated through (45). If a set $B$ of more than one estimator in the bank provides a reliable estimation of a fault $f_{i}$, we define $\hat{f}_{i}$ through the reliable estimator with better performance w.r.t. certain isolation performance index

$$
\hat{f}_{i}(k):=\left\{\hat{f}_{l}^{b^{*}}(k): i=\pi_{l}^{b^{*}}\right\},
$$

with

$$
b^{*}=\left\{\underset{b \in B}{\operatorname{argmin}} \alpha_{l}^{b}: \pi_{l}^{b}=i\right\},
$$

and $\alpha_{l}^{b}$ certain isolation performance index reflecting and improved isolation performance as it decreases.

\footnotetext{
${ }^{9}$ Once a fault is accommodated, the observers in the bank must be reset to avoid the existence of wrong initial conditions derived from the previous presence of ignored faults.
} 


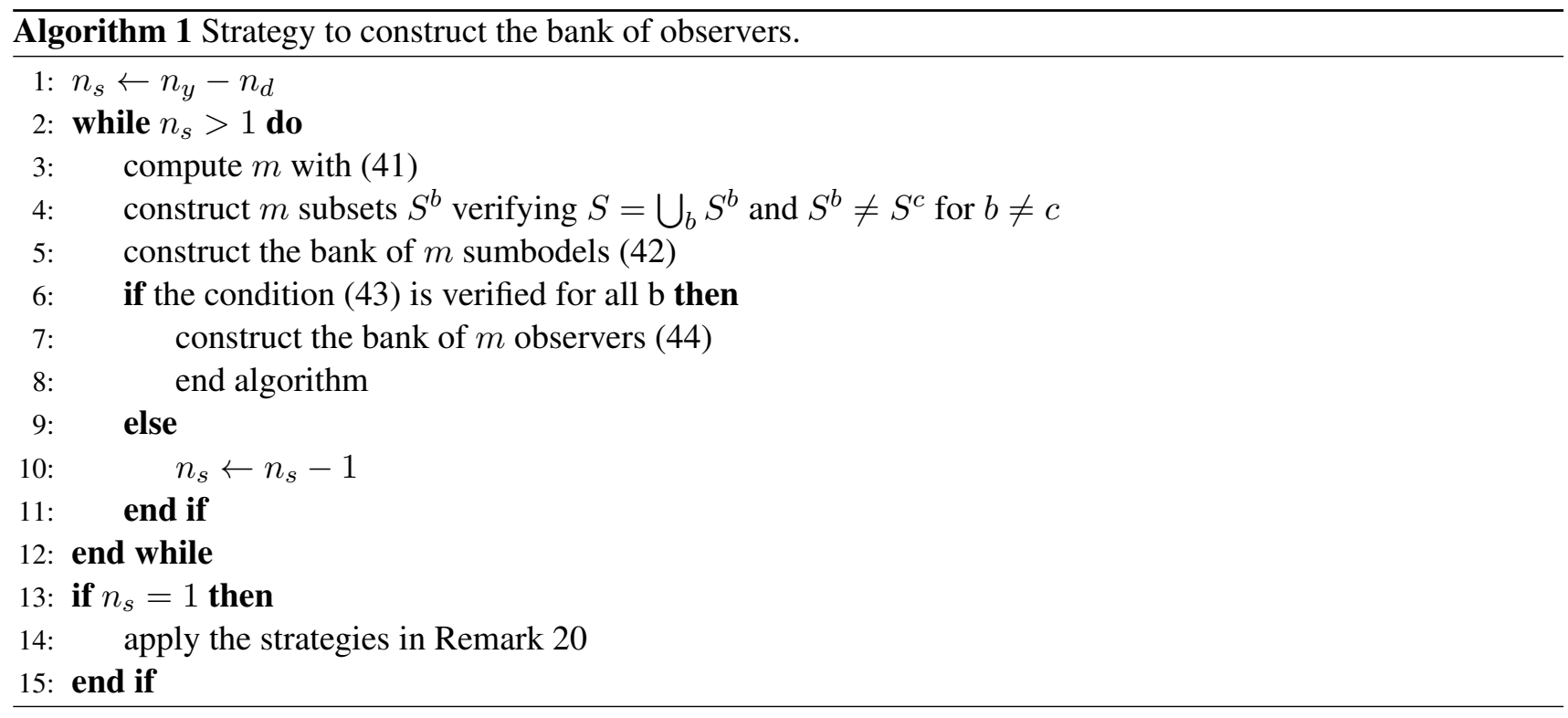

\subsection{FI Performance Characterization and Co-design with FI Requirements}

The isolation index $\mathbf{i} . \mathbf{1}$ of a fault $i, \phi_{i}$, depends on the false alarms of every pair $\hat{f}_{l}^{b}$ and $J_{l}^{b}$ with $\pi_{l}^{b}=i$, i.e.,

$$
\phi_{i}=\mathbb{P}\left\{\bigcap_{(b, l): \pi_{l}^{b}=i} \exists k:\left|\hat{f}_{l}^{b}(k)\right| \geq J_{l}^{b}\right\},
$$

when $f_{i}=0$. Note that the events $X_{j}=$ " $\exists k:\left|\hat{f}_{l}^{b}(k)\right| \geq J_{l}^{b}$ " in (47) (with $j=1, \ldots, n_{j}$ and $n_{j}$ the number of pairs $(b, l)$ satisfying $\pi_{l}^{b}=i$ ) are not independent and $\phi_{i}$ depends on the conditional probability of each event $X_{j}$ subject to the occurrence of the others. Starting from event $X_{1}$, we have that

$$
\phi_{i}=\mathbb{P}\left\{X_{1}\right\} \cdot \mathbb{P}\left\{X_{2} / X_{1}\right\} \cdot \ldots \cdot \mathbb{P}\left\{X_{n_{j}} / X_{1} \cap \ldots \cap X_{n_{j}-1}\right\} .
$$

This equality holds when starting from any event $X_{j}$ and, thus, we have that

$$
\phi_{i} \leq \mathbb{P}\left\{X_{j}\right\}
$$

for $j=1, \ldots, n_{j}$. The conditional probabilities in (48) are close to 1 because, in practice, the same noises affect all the observers simultaneously. Hence, we deduce that $\phi_{i}$ is tightly bounded by

$$
\phi_{i} \leq \min _{(b, l): \pi_{l}^{b}=i} \phi_{l}^{b}
$$

where $\phi_{l}^{b}$ satisfies (27) for the $b$-th estimator. Regarding the index i.1, the minimum isolable constant fault $i, \psi_{i}$, is given by

$$
\psi_{i}=\max _{(b, l): \pi_{l}^{b}=i} \psi_{l}^{b},
$$

where $\psi_{l}^{b}$ satisfies (28) for the $b$-th estimator. Analogous definitions apply to the time indices i.3 and i.4.

In order to design the observers and decision mechanisms of the bank for satisfying global isolation performance requirements, we make use of these characterizations. The following two strategies show a proposal of how to perform the co-design of each observer and the corresponding thresholds for guaranteeing different isolation requirements.

Strategy 5. Assume that we desire to ensure altogether certain false isolation rates $\phi_{i}^{*}\left(i=1, \ldots, n_{f}\right)$, certain minimum isolable constant faults $\psi_{i}^{*}\left(i=1, \ldots, n_{f}\right)$ and minimum acknowledgement times under ramp faults. 
To ensure these requirements, we perform $m$ independent designs. In each of them, we design a different observer $b$ of the bank and the corresponding thresholds $J_{l}^{b}\left(l=1, \ldots, n_{s}\right)$ through Strategy 3 with requirements $\phi_{i}^{*}$ and $\psi_{i}^{*}$ whenever $\pi_{l}^{b}=i$.

Strategy 6. Assume that we desire to ensure altogether certain false isolation rates $\phi_{i}^{*}\left(i=1, \ldots, n_{f}\right)$, certain acknowledgement times under ramp faults $\vartheta_{i}^{*}\left(i=1, \ldots, n_{f}\right)$ and we want to minimise the minimum isolable constant faults. To ensure these requirements, we perform $m$ independent designs. In each of them, we design a different observer $b$ of the bank and the corresponding thresholds $J_{l}^{b}\left(l=1, \ldots, n_{s}\right)$ through Strategy 4 with requirements $\phi_{i}^{*}$ and $\vartheta_{i}^{*}$ whenever $\pi_{l}^{b}=i$.

\section{Case of Study: FE and FI in a Wind Turbine}

The benchmark in (Odgaard et al., 2013) describes a three-bladed horizontal wind turbine which consists of four main systems: the generator and converter, the drive train, the blade and pitch and the controller. The strategies developed in this paper are independent of the control scheme and can be implemented regardless of the control law. In this section, we apply the proposed fault estimators and isolators to the first three systems.

\subsection{State Space Models}

In the following, we model the wind turbine systems through the continuous model

$$
\begin{aligned}
\dot{x} & =A^{c} x+B^{c} u+E^{c} f+G^{c} v+D^{c} d, \\
y & =C^{c} x+F^{c} f+H^{c} v .
\end{aligned}
$$

The sate-space matrices of the realizations are detailed in Appendix B. Reference (Odgaard et al., 2013), which is referred for further modelling details, specifies that the noises that affect the wind turbine benchmark are Gaussian.

Generator and Converter System. This system can be modelled as a first order closed-loop system between the torque reference, $\tau_{g, r}$, and the non-deviated torque $\tau_{g, n}$. The actual generator torque, $\tau_{g}$, is given by $\tau_{g}=$ $\tau_{g, n}+\Delta \tau_{g, n}$, where $\Delta \tau_{g, n}$ is the offset representing the converter fault. Let $\tau_{g, m}$ and $v_{\tau_{g}}$ be the measurement of $\tau_{g}$ and the corresponding additive noise; then, we have

$$
x \triangleq \tau_{g, n}, \quad u \triangleq \tau_{g, r}, \quad y \triangleq \tau_{g, m}, \quad f \triangleq \Delta \tau_{g, n}, \quad v \triangleq v_{\tau_{g}}, \quad d \triangleq \emptyset .
$$

Drive Train System. The drive train dynamics is represented by a two-mass model involving the rotor speed, $\omega_{r}$, the generator speed, $\omega_{g}$ and the torsion angle of the drive train, $\theta_{r g}$. This system is fed with the actual generator torque, $\tau_{g}$, and the aerodynamic torque from the wind, $\tau_{a}$. Provided that the real generator torque is not available, we model this input as the difference between its measurement, $\tau_{g, m}$, and the corresponding additive sensor noise, $v_{\tau_{g, m}}$. The aerodynamic torque may be obtained through the wind speed and the power coefficient, $C_{p}$, which is a nonlinear function of $\omega_{r}$, the wind speed and the pitch angles of the turbine. In practice, it is very difficult to know the real distribution of $C_{p}$ and the measurements of the wind speed provided by anemometers are rather inaccurate. Thus, we consider $\tau_{a}$ to be an UI, which is a widely extended assumption in the bibliography, see (Odgaard, Stoustrup, Nielsen, \& Damgaard, 2009). Both drive train speeds are measured by the redundant sensors $\omega_{r, m_{1}}, \omega_{r, m_{2}}, \omega_{g, m_{1}}$ and $\omega_{g, m_{2}}$ and we model their possible faults as the additive signals $\Delta \omega_{r, m_{1}}, \Delta \omega_{r, m_{2}}$, $\Delta \omega_{g, m_{1}}$ and $\Delta \omega_{g, m_{2}}$. Similar applies to their corresponding sensor noise. In all, the state-space vectors are

$$
\begin{aligned}
& x \triangleq\left[\begin{array}{lll}
\omega_{r} & \omega_{g} & \theta_{r g}
\end{array}\right]^{T}, \\
& u \triangleq \tau_{g, m}, \\
& y \triangleq\left[\begin{array}{llll}
\omega_{r, m_{1}} & \omega_{r, m_{2}} & \omega_{g, m_{1}} & \omega_{g, m_{2}}
\end{array}\right]^{T}, \\
& v \triangleq\left[\begin{array}{lllll}
v_{\omega_{r, m_{1}}} & v_{\omega_{r, m_{2}}} & v_{\omega_{g, m_{1}}} & v_{\omega_{g, m_{2}}} & v_{\tau_{g, m}}
\end{array}\right]^{T}, \\
& f \triangleq\left[\begin{array}{llll}
\Delta \omega_{r, m_{1}} & \Delta \omega_{r, m_{2}} & \Delta \omega_{g, m_{1}} & \Delta \omega_{g, m_{2}}
\end{array}\right]^{T}, \\
& d \triangleq \tau_{a} \text {. }
\end{aligned}
$$




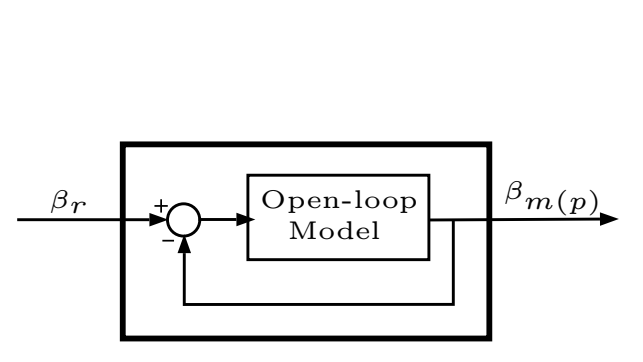

Available Closed-loop Model

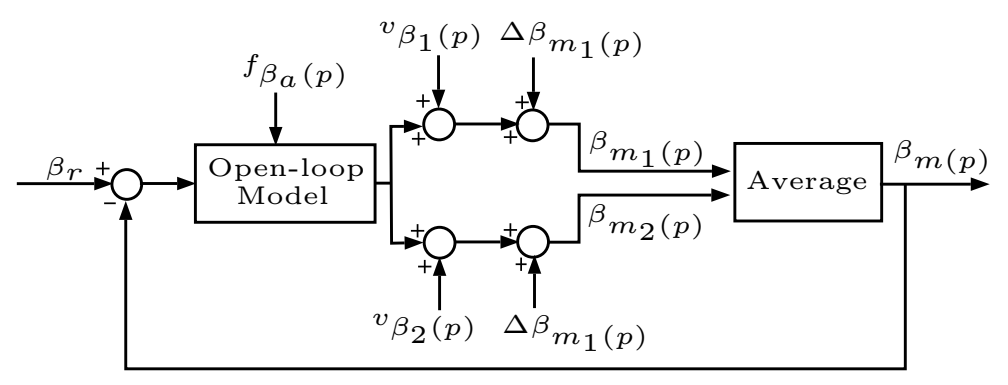

Actual Feedback Implementation Model

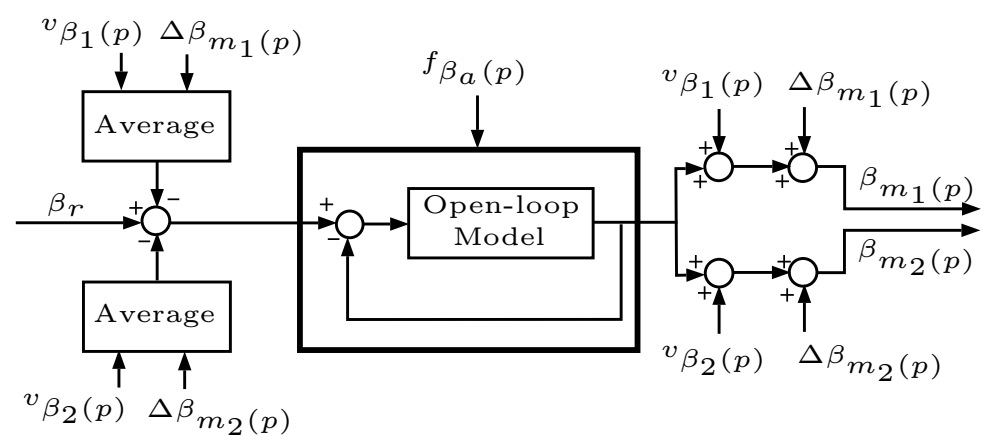

Equivalent Feedback Implementation Model

Figure 2: Architecture of the pitch system.

Blade and Pitch System. The hydraulic pitch system of each of the blades $p=1,2,3$ is modelled as a second order closed-loop system between the reference angle provided by the wind turbine controller, $\beta_{r}$, and the averaged measurement, $\beta_{m(p)}$, provided by two redundant sensors, $\beta_{m_{1}(p)}$ and $\beta_{m_{2}(p)}$. Both sensors entail measurement noises that disturb the closed loops. Provided that only the closed-loop indices $\left(\omega_{n_{0}}\right.$ and $\left.\xi_{0}\right)$ are known, we model these disturbances as additive signals $v_{\beta_{1(p)}}$ and $v_{\beta_{2(p)}}$ which affect both the measurements and the reference. Similar applies to sensor faults, which we denote as $\Delta \beta_{m_{1}(p)}$ and $\Delta \beta_{m_{2}(p)}$ (See Fig.2).

The pitch actuator may also suffer from dynamic changes. If we denote the deviations of $\omega_{n_{0}}$ and $\xi_{0}$ as $\Delta \omega_{n(p)}$ and $\Delta \xi_{(p)}$ (i.e., $\omega_{n(p)}=\omega_{n_{0}}+\Delta \omega_{n(p)}$ and $\left.\xi_{(p)}=\xi_{0}+\Delta \xi_{(p)}\right)$, the actuator fault can be modelled as an additive signal in the form of

$$
f_{\beta_{a}(p)}=\left(\Delta w_{n(p)}^{2}+2 w_{n_{0}} \Delta w_{n(p)}\right)\left(\beta_{r}-\beta_{(p)}\right)-2\left(\xi_{0} \Delta w_{n(p)}+w_{n_{0}} \Delta \xi_{(p)}+\Delta \xi_{(p)} \Delta w_{n(p)}\right) \dot{\beta}_{(p)} .
$$

In all, the state-space vectors of each pitch system are

$$
\begin{aligned}
& x \triangleq\left[\begin{array}{ll}
\beta_{(p)} & \dot{\beta}_{(p)}
\end{array}\right]^{T}, \\
& u \triangleq \beta_{r} \text {, } \\
& y \triangleq\left[\begin{array}{ll}
\beta_{m_{1}(p)} & \beta_{m_{2}(p)}
\end{array}\right]^{T}, \\
& v \triangleq\left[\begin{array}{ll}
v_{\beta_{1(p)}} & v_{\beta_{2(p)}}
\end{array}\right]^{T}, \\
& f \triangleq\left[\begin{array}{lll}
f_{\beta_{a}(p)} & \Delta \beta_{m_{1}(p)} & \Delta \beta_{m_{2}(p)}
\end{array}\right]^{T}, \\
& d \triangleq \emptyset \text {. }
\end{aligned}
$$


Table 4: Fault signature matrix in the drive train system ( $\otimes:$ Sensitive, $\square$ :Ignored).

\begin{tabular}{|c|c|c|c|c|c|c|c|c|c|c|c|c|}
\hline & $\hat{f}_{1}^{1}$ & $\hat{f}_{2}^{1}$ & $\hat{f}_{3}^{1}$ & $\hat{f}_{1}^{2}$ & $\hat{f}_{2}^{2}$ & $\hat{f}_{3}^{2}$ & $\hat{f}_{1}^{3}$ & $\hat{f}_{2}^{3}$ & $\hat{f}_{3}^{3}$ & $\hat{f}_{1}^{4}$ & $\hat{f}_{2}^{4}$ & $\hat{f}_{3}^{4}$ \\
\hline$f_{1}=\Delta \omega_{r, m_{1}}$ & $\nabla$ & & & $\nabla$ & & & $\nabla$ & & & $\square$ & $\square$ & $\square$ \\
\hline$f_{2}=\Delta \omega_{r, m_{2}}$ & & $\otimes$ & & & $\nabla$ & & $\square$ & $\square$ & $\square$ & $\otimes$ & & \\
\hline$f_{3}=\Delta \omega_{g, m_{1}}$ & & & $\otimes$ & $\square$ & $\square$ & $\square$ & & $\otimes$ & & & 又 & \\
\hline$f_{4}=\Delta \omega_{g, m_{2}}$ & $\square$ & $\square$ & $\square$ & & & 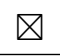 & & & $\otimes$ & & & $凶$ \\
\hline
\end{tabular}

Table 5: Fault signature matrix in the pitch systems ( :Sensitive, $\square:$ Ignored).

\begin{tabular}{|l|cc|cc|cc|}
\cline { 2 - 6 } \multicolumn{1}{c|}{} & $\hat{f}_{1}^{1}$ & $\hat{f}_{2}^{1}$ & $\hat{f}_{1}^{2}$ & $\hat{f}_{2}^{2}$ & $\hat{f}_{1}^{3}$ & $\hat{f}_{2}^{3}$ \\
\hline$f_{1}=f_{\beta_{a}(p)}$ & $\bigotimes$ & & $\bigotimes$ & & $\square$ & $\square$ \\
$f_{2}=\Delta \beta_{m_{1}(p)}$ & & $\bigotimes$ & $\square$ & $\square$ & $\bigotimes$ & \\
$f_{3}=\Delta \beta_{m_{2}(p)}$ & $\square$ & $\square$ & & $\bigotimes$ & & $\square$ \\
\hline
\end{tabular}

\subsection{Fault Estimation and Isolation}

\subsubsection{FE and FI Architecture}

We discretise the models (51) with the sample time $T_{s}=0.01 \mathrm{~s}$ and we obtain the matrices $A, B, C, D, E, F$, $G$ and $H$ in (1). The converter faults fulfil the necessary condition for fault isolability in Assumption 4; however, the drive train and the pitch faults do not verify it. Then, we split these two models as detailed in Section 5. For the drive train system, the strategy summarised in Algorithm 1 leads to $n_{s}=3$ and $m=4$ :

$$
\begin{gathered}
S^{1}=\left\{f_{1}, f_{2}, f_{3}\right\}, \quad S^{2}=\left\{f_{1}, f_{2}, f_{4}\right\}, \quad S^{3}=\left\{f_{1}, f_{3}, f_{4}\right\}, \quad S^{4}=\left\{f_{2}, f_{3}, f_{4}\right\}, \\
\pi^{1}=\{1,2,3\}, \quad \pi^{2}=\{1,2,4\}, \quad \pi^{3}=\{1,3,4\}, \quad \pi^{4}=\{2,3,4\}
\end{gathered}
$$

then, FI is only guaranteed if no more than two simultaneous faults occur. For each pitch system, the strategy summarised in Algorithm 1 indicates that the procedure in Remark 20 must be applied. We get $n_{s}=2$ and $m=3$ :

$$
\begin{gathered}
S^{1}=\left\{f_{1}, f_{2}\right\}, \quad S^{2}=\left\{f_{1}, f_{3}\right\}, \quad S^{3}=\left\{f_{2}, f_{3}\right\}, \\
\pi^{1}=\{1,2\}, \quad \pi^{2}=\{1,3\}, \quad \pi^{3}=\{2,3\} .
\end{gathered}
$$

The isolability condition (43) holds for the subsets $S^{1}$ and $S^{2}$, and all the faults of the pitch system are considered within these subsets. For the subset $S^{3}$ the condition (43) does not hold and we must use $A_{F}^{b}=0, B_{F}^{b}=I$, $C_{F}^{b}=I$ to extend this submodel.

For each of the resulting submodels, we define the observers (44) and the corresponding decision mechanisms (45). The signature matrices are presented in Table 4 and Table 5, where $\nabla$ indicates that the estimate $f_{l}^{b}$ is devoted to the estimation of the fault $f_{i}$ and $\square$ indicates that the fault $f_{i}$ is ignored by the observer $b$ and it may corrupt the estimates $f_{l}^{b}$ with $l=1, \ldots, n_{s}$. For each of the resulting submodels, we define the observers (44) and the corresponding decision mechanisms (45).

\subsubsection{FE and FI Design}

Let us first perform different observer designs to study the existing trade-offs between the estimation performance criteria detailed in Section 3.1 ${ }^{10}$. Fig.3 (left) shows the estimation performance results for different observers designed through Strategy 1 for the converter system. One verifies that imposing more restrictive constraints over the ramp fault tracking delay $T_{11}$ leads to higher marginal variances due to noises $\Sigma_{11}$. Fig.4 includes the details on the frequency response of the closed-loop transfer function between $f$ and $\hat{f}$ for some of these observers (Observer A with $T_{11}=1$ samples, Observer B with $T_{11}=3$ samples and Observer $\mathrm{C}$ with $T_{11}=5$ samples). Provided the physical proprieties of the converter system, the transfer function between $f$ and $\hat{f}$ coincides with

\footnotetext{
${ }^{10}$ The problems are set up in YALMIP (Lofberg, 2004) and we successfully solve them with the PENBMI solver (Henrion et al., 2005). For sake of brevity, we do not include the value of the obtained gain matrices.
} 

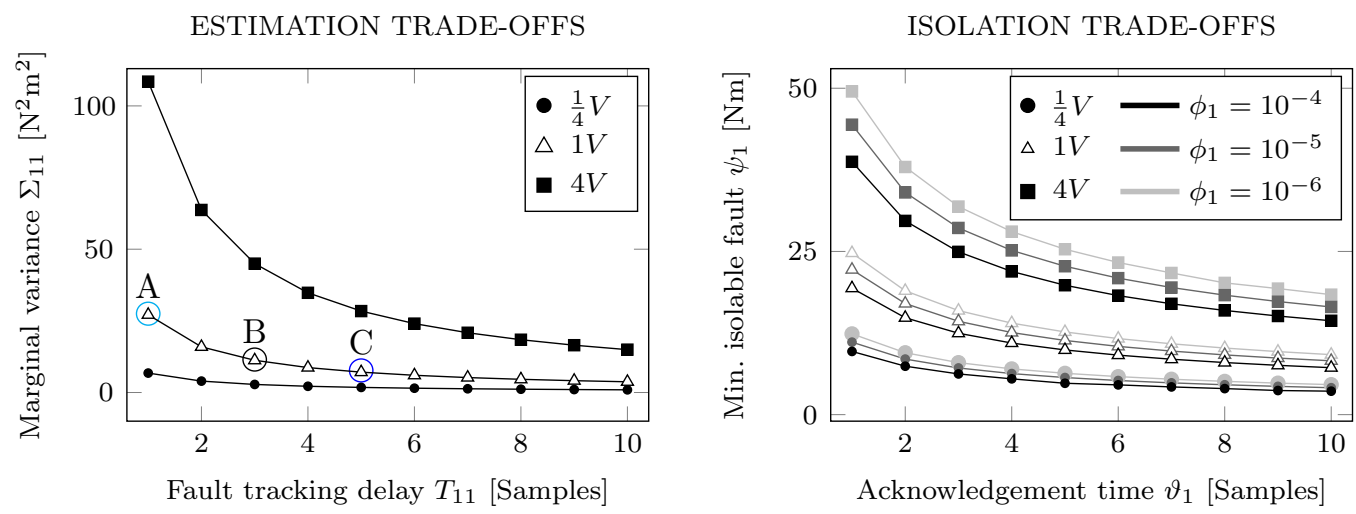

Figure 3: Estimation and isolation performance trade-offs in the converter system.

the transfer function between $v$ and $\hat{f}$. One verifies then that the observers with a higher bandwidth and a lower phase lag (i.e., fastest response under the appearance of faults) are characterized by higher magnitudes at high frequencies (i.e., higher noise influence). Fig.3 (left) also represents the effect of performing these designs in situations of amplified (i.e., $4 \mathrm{~V}$ ) and attenuated (i.e., $1 / 4 \mathrm{~V}$ ) noises, where $\mathrm{V}$ denotes the noise covariance in the benchmark. When the noises affecting the systems increase in variance, the same fault tracking delays imply higher variances.

Now, we perform the observer and decision mechanism co-design in Strategy 4 with different acknowledgement time requirements. Fig.3 (right) depicts the trade-offs between the isolation performance indices defined in Section 4.1. Again, imposing more restrictive time constraints leads to higher minimum isolable constant faults for certain level of false alarms. For its part, increasing the false isolation rate reduces the value of the minimum isolable constant faults for certain acknowledgement time of ramp faults.

To fulfil the requirements in the benchmark (Odgaard et al., 2013), we now design the observers and decision mechanisms of the three wind turbine systems through Strategy 4. Although the benchmark (Odgaard et al., 2013) highlights the necessity of isolating the faults occurring in the wind turbine systems, it only explicitly specifies requirements over detection performance indices. Thus, we equal the requirements over the false detection rates and the detection times in the benchmark to the requirements over the false isolation rates and the isolation times, respectively. In order to directly apply our approach, we approximate the requirements over the isolation times to requirements over the acknowledgement times of ramp faults and we perform the co-design of each pair of observer and mechanism in the banks. In number of samples, we have $\vartheta_{1}^{*}=3$ for the converter, $\vartheta_{\{1,2,3,4\}}^{*}=10$ for the drive train system, and $\vartheta_{1}^{*}=8, \vartheta_{\{2,3\}}^{*}=10$ for the pitch system. The required false isolation rate is $\phi_{i}^{*}=10^{-5}$ for all the systems. We use the summation (i.e., $\left.\sum_{l=1}^{n_{s}} \Xi_{l l}\right)$ as the function $f\left(\Xi_{11}, \ldots, \Xi_{n_{s} n_{s}}\right)$ being minimised in the optimization problem. The obtained minimum isolable constant faults are indicated in Table 6.

\subsection{Simulation Results}

The wind turbine benchmark presents an scenario of $4400 \mathrm{~s}$ in which different faults occur. Within the listing of the possible faults, the benchmark test sequences choose the subset of faults in Table 7 . The benchmark considers seven different test signal sets; they are formed by time-shifting the occurrence of the faults defined in the original

Table 6: Minimum isolable constant faults.

\begin{tabular}{cc}
\hline Fault & Minimum Isolable Fault \\
\hline$\Delta \omega_{r, m_{1}}$ & $0.151 \mathrm{rad} / \mathrm{s}$ \\
$\Delta \omega_{r, m_{2}}$ & $0.151 \mathrm{rad} / \mathrm{s}$ \\
$\Delta \omega_{g, m_{1}}$ & $0.295 \mathrm{rad} / \mathrm{s}$ \\
$\Delta \omega_{g, m_{2}}$ & $0.295 \mathrm{rad} / \mathrm{s}$ \\
\hline
\end{tabular}

\begin{tabular}{cc}
\hline Fault & Minimum Isolable Fault \\
\hline$\Delta \tau_{g, n}$ & $14.300 \mathrm{Nm}$ \\
$f_{\beta_{a}(p)}$ & $0.933^{\circ} / s^{2}$ \\
$\Delta \beta_{m_{1}(p)}$ & $0.063^{\circ}$ \\
$\Delta \beta_{m_{2}(p)}$ & $0.063^{\circ}$ \\
\hline
\end{tabular}



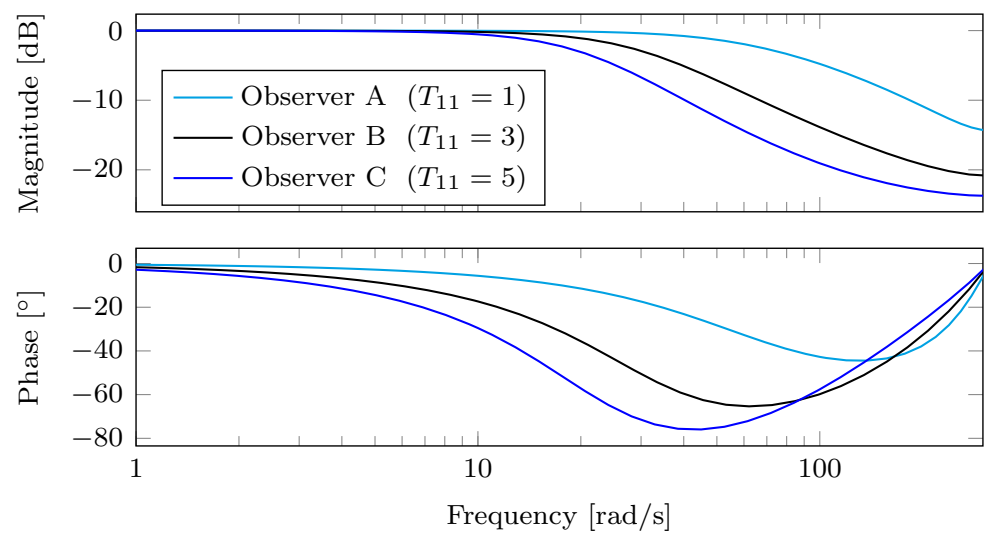

Figure 4: Frequency response of the closed-loop transfer function between $f$ and $\hat{f}$ (Converter system).

Table 7: Wind turbine benchmark fault scenario description.

\begin{tabular}{ccccc}
\hline Fault code & System & Fault signal & Fault type & Time occurrence TS1 \\
\hline GC-1 & Generator & $\Delta \tau_{g, n}$ & offset & $t \in[3800,3900] \mathrm{s}$ \\
DT-1 & Drive Train & $\Delta \omega_{r, m_{1}}$ & fixed value & $t \in[1500,1600] \mathrm{s}$ \\
DT-2 & Drive Train & $\Delta \omega_{r, m_{2}}, \Delta \omega_{g, m_{1}}$ & gain factor & $t \in[1000,1100] \mathrm{s}$ \\
P1-1 & Pitch 1 & $\Delta \beta_{m_{1}(1)}$ & fixed value & $t \in[2000,2100] \mathrm{s}$ \\
P2-1 & Pitch 2 & $\Delta \beta_{m_{2}(2)}$ & gain factor & $t \in[2300,2400] \mathrm{s}$ \\
P2-2 & Pitch 2 & $f_{\beta_{a}(2)}$ & change dynamics & $t \in[2900,3000] \mathrm{s}$ \\
P3-1 & Pitch 3 & $\Delta \beta_{m_{1}(3)}$ & fixed value & $t \in[2600,2700] \mathrm{s}$ \\
P3-2 & Pitch 3 & $f_{\beta_{a}(3)}$ & change dynamics & $t \in[3400,3500] \mathrm{s}$ \\
\hline
\end{tabular}
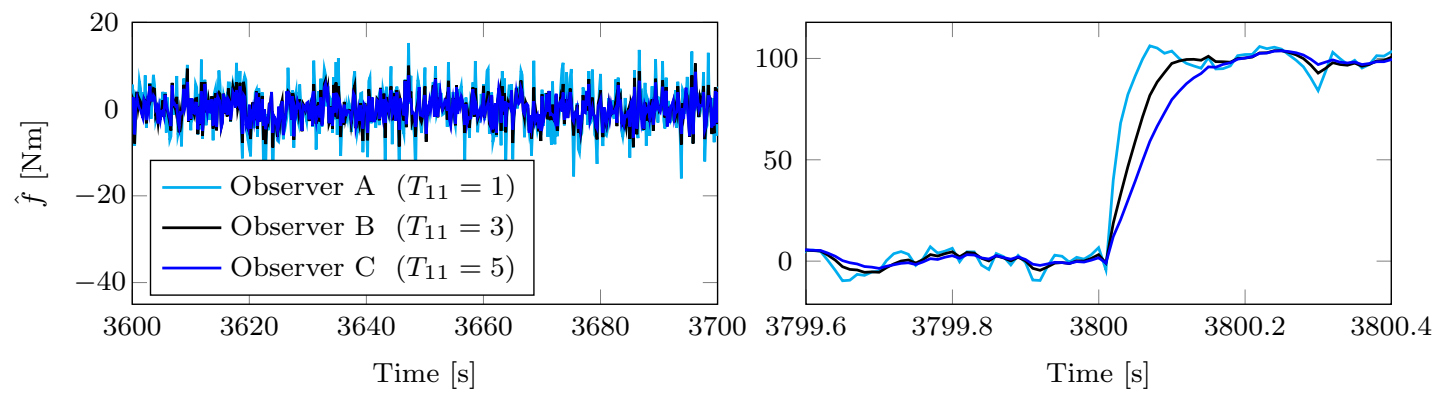

Figure 5: FE in the converter with different observers (test set TS1).

test sequence (TS1), which is described in Table 7.

Illustratively, let us first simulate the time response of the Observers A, B and C defined in Fig.4 to estimate the converter fault in the test set TS1. Fig.5 (left) shows the estimation results in fault-free samples and Fig.5 (right) shows the estimation results during the fault appearance. The simulation results validate the estimation trade-offs indicated in Fig.3 (left).

Let us also analyse the effect of the restrictiveness of the time constraints on the sensitivity to parameter changes in the model. Fig.6 shows the effect produced by a 5\% relative change in the parameter defining the converter dynamics. We verify that the sensitivity to parameter changes increases as the time constraints become more restrictive. The reader is referred to Remark 3 and equation (7) to obtain the algebraic expression of the effect of the parameter changes on the fault estimation error ${ }^{11}$.

\footnotetext{
${ }^{11}$ If the uncertainties regarding parameter changes lead to poor estimation results, these uncertainties must be modelled as UIs (see Remark 3) and certain degree of UI decoupling (i.e., constraint (19)) must be introduced as an additional requirement in the observer design.
} 


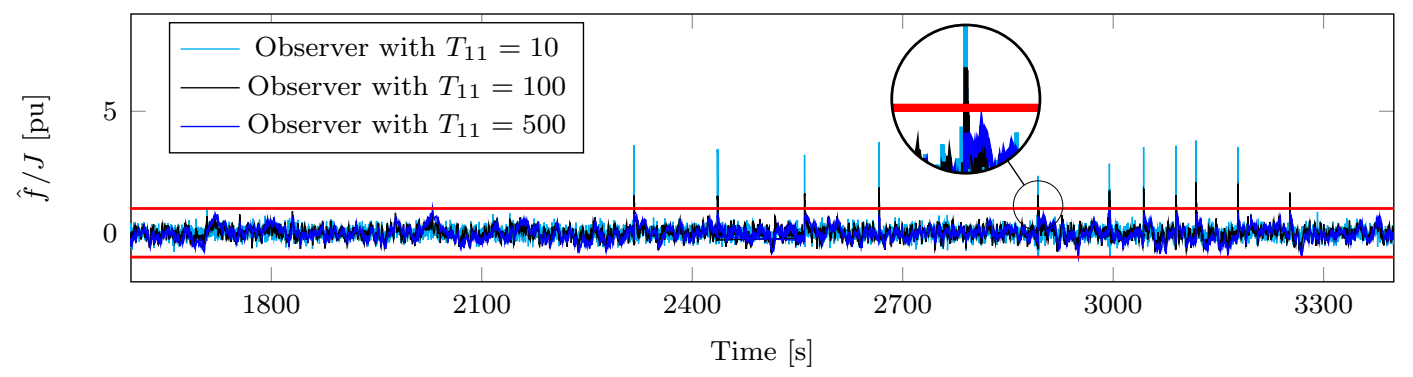

Figure 6: FE in the converter with model parameter changes (test set TS1).

Table 8: Isolation times (IT) in number of samples of the Monte Carlo simulation.

\begin{tabular}{ccccc}
\hline Fault scenario & IT Requirement & Minimum IT & Mean IT & Maximum IT \\
\hline GC-1 & 3 & 2 & 2.19 & 3 \\
DT-1 & 10 & 2 & 4.36 & 11 \\
DT-2 & 10 & 2 & 2 & 2 \\
P1-1 & 10 & 2 & 2 & 2 \\
P2-1 & 10 & 6 & 223.8 & 737 \\
P2-2 & 8 & 2 & 2.56 & 7 \\
P3-1 & 10 & 2 & 2.46 & 13 \\
P3-2 & 8 & 1145 & 1793.4 & 2322 \\
\hline
\end{tabular}

Now, we test the behaviour of the observers and decision mechanics that we have designed with the isolation performance constraints in the benchmark. If we simulate the different test sets proposed in the benchmark through several Monte Carlo simulations for different noises, we verify that all the results verify the false isolation rate restriction tightly. The isolation times of the faults are summarised in Table 8. As an example, Fig. 7 details the isolation times obtained for the fault DT1-1. Note that the minimum isolation times fulfil the requirements in the benchmark. The cases in which the time requirement is exceeded refer to scenarios with variable fault signals which do not always exceed the achieved minimum isolable fault. For instance, there are cases in which the fault P3-2 is present in the system but the pitch reference is barely zero. In such cases, there is no chance to detect or isolate the changes experienced by the pitch dynamics. Other proposals available in the bibliography provide similar results regarding this issue. In any case, if the designer decides that missisolating these small faults may be prohibitive, it would be possible to redesign the fault isolators through Strategy 5 as explained in Section 5 . Note that numerical comparisons with other strategies in the literature are difficult because most existing works are devoted to fault detection and fault isolation and estimation are not included. Moreover, they study indices as the FDR instead of the physically meaningful parameters required on the benchmark (i.e., isolation times, minimum isolable faults, etc.).

In the following, we include the figures showing the FE and FI results in the test set TS1 (described in Table 7). First, Fig.8 shows the estimation signal and the corresponding isolation threshold for the converter system, which is affected by GC-1. Fig. 9 shows the outputs provided by the bank built for FE and FI in the drive train system, which is affected by DT-1 and DT-2. It is straightforward to verify that applying (45)-(46), the isolation and the estimation of the faults DT-1 and DT-2 is achieved.

Regarding the pitch system, Fig.10 (details in Fig. 11) shows the results for the third pitch system, which is affected by both P3-1 and P3-2. The figure includes the fault estimates and the thresholds corresponding to the relied observers in the bank. Note that the observer which provides the estimate of the fault $f_{3}=\Delta \beta_{m_{2}(p)}$ (i.e., the pair with better isolation optimised performance index) becomes non-reliable when $f_{2}=\Delta \beta_{m_{1}(p)}$ is present in the system. In this case, the estimation is provided by another pair in the bank with a poorer minimum isolable fault. For ease of space, we do not include the results for the first and second pitch systems. 


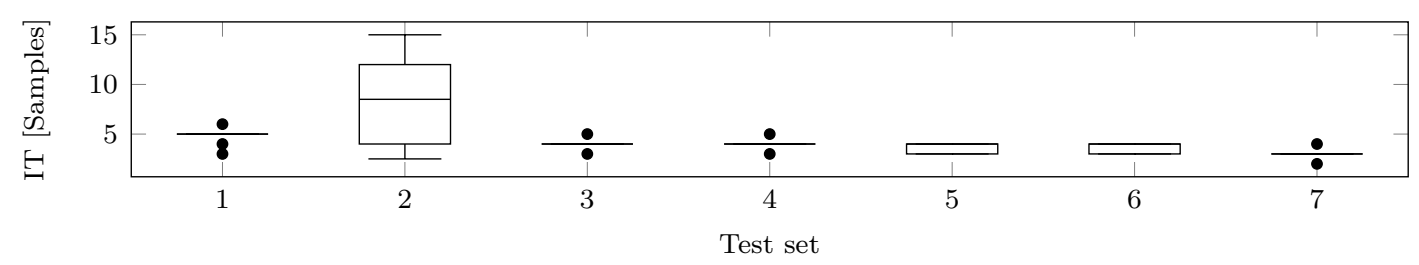

Figure 7: Isolation time (IT) of the fault DT-1 for the test sets in the benchmark.
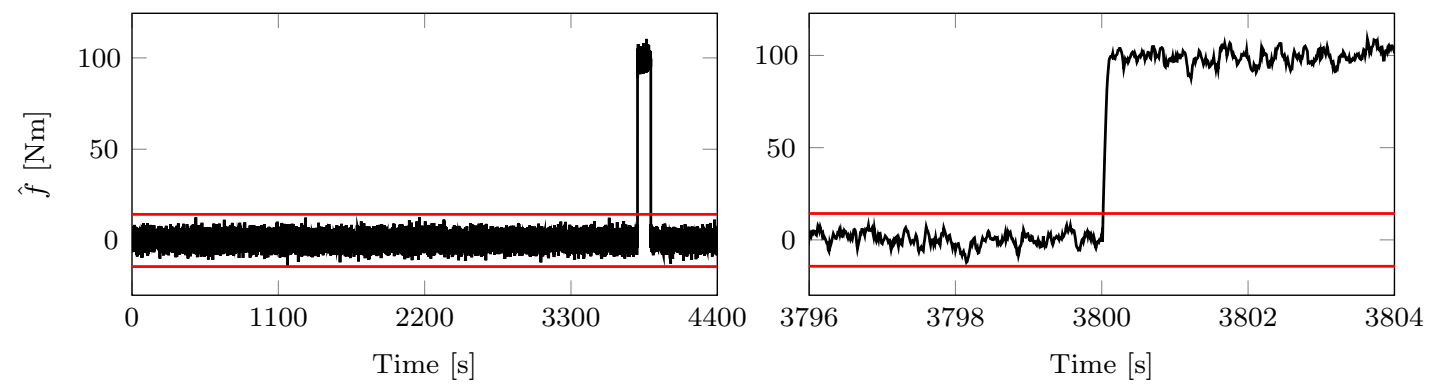

Figure 8: FE and FI in the converter (test set TS1).
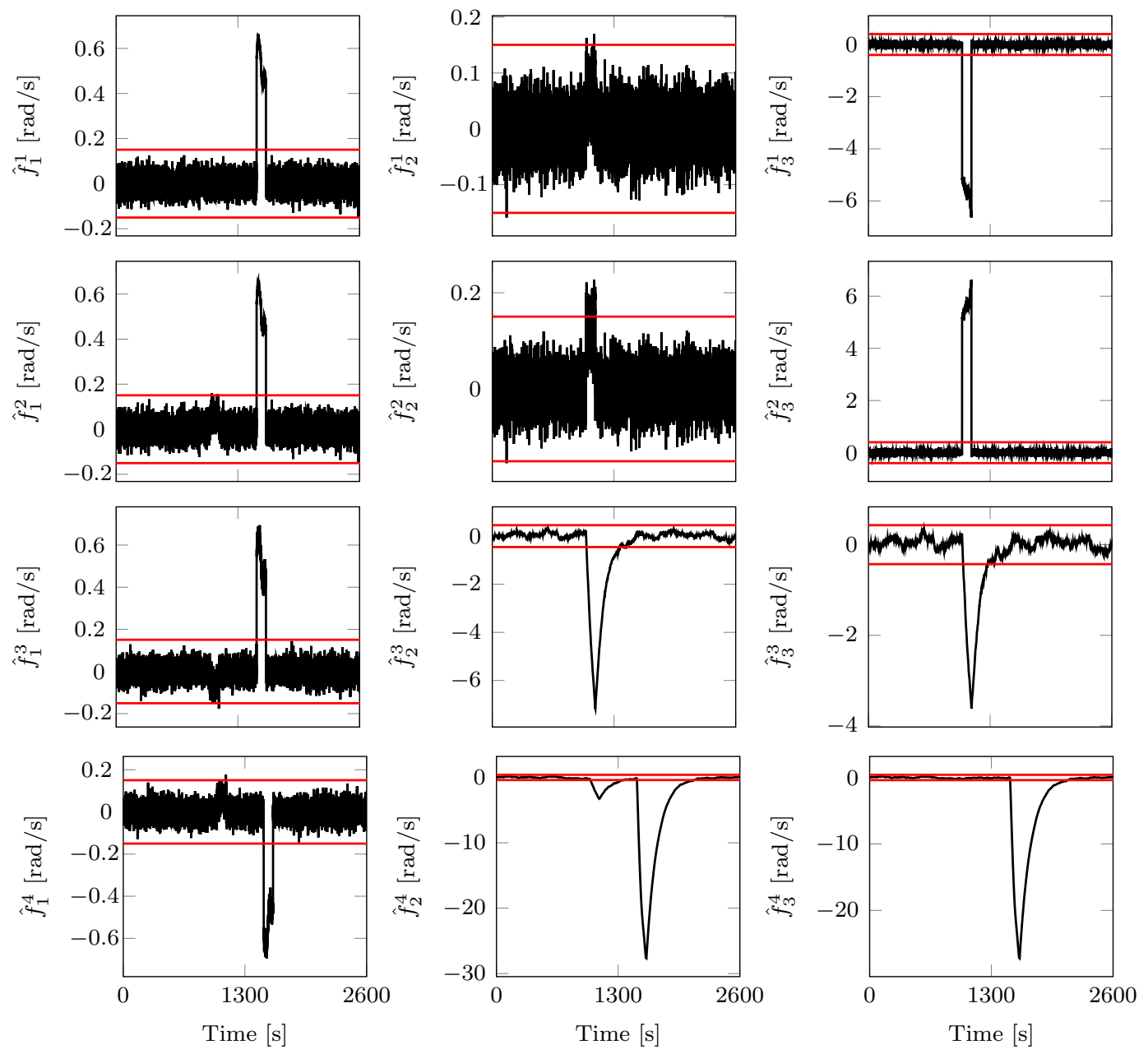

Figure 9: Bank of observers and decision mechanisms in the drive train (test set TS1). 

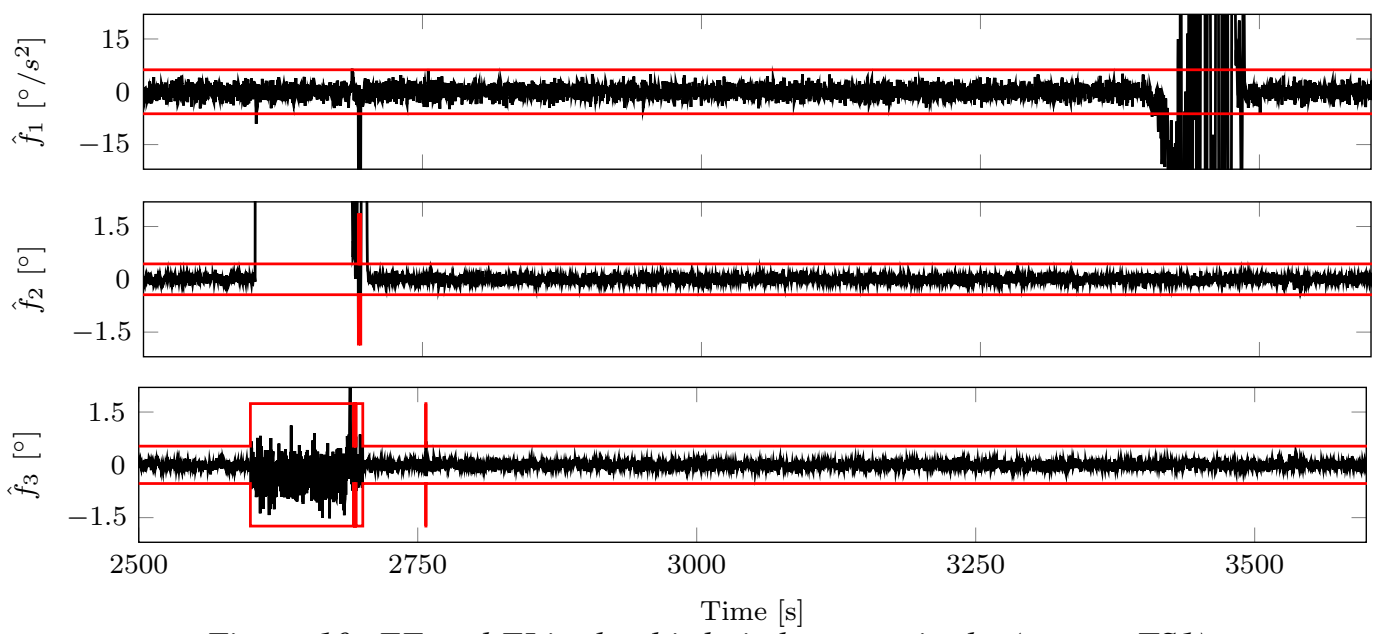

Figure 10: FE and FI in the third pitch system in the (test set TS1).
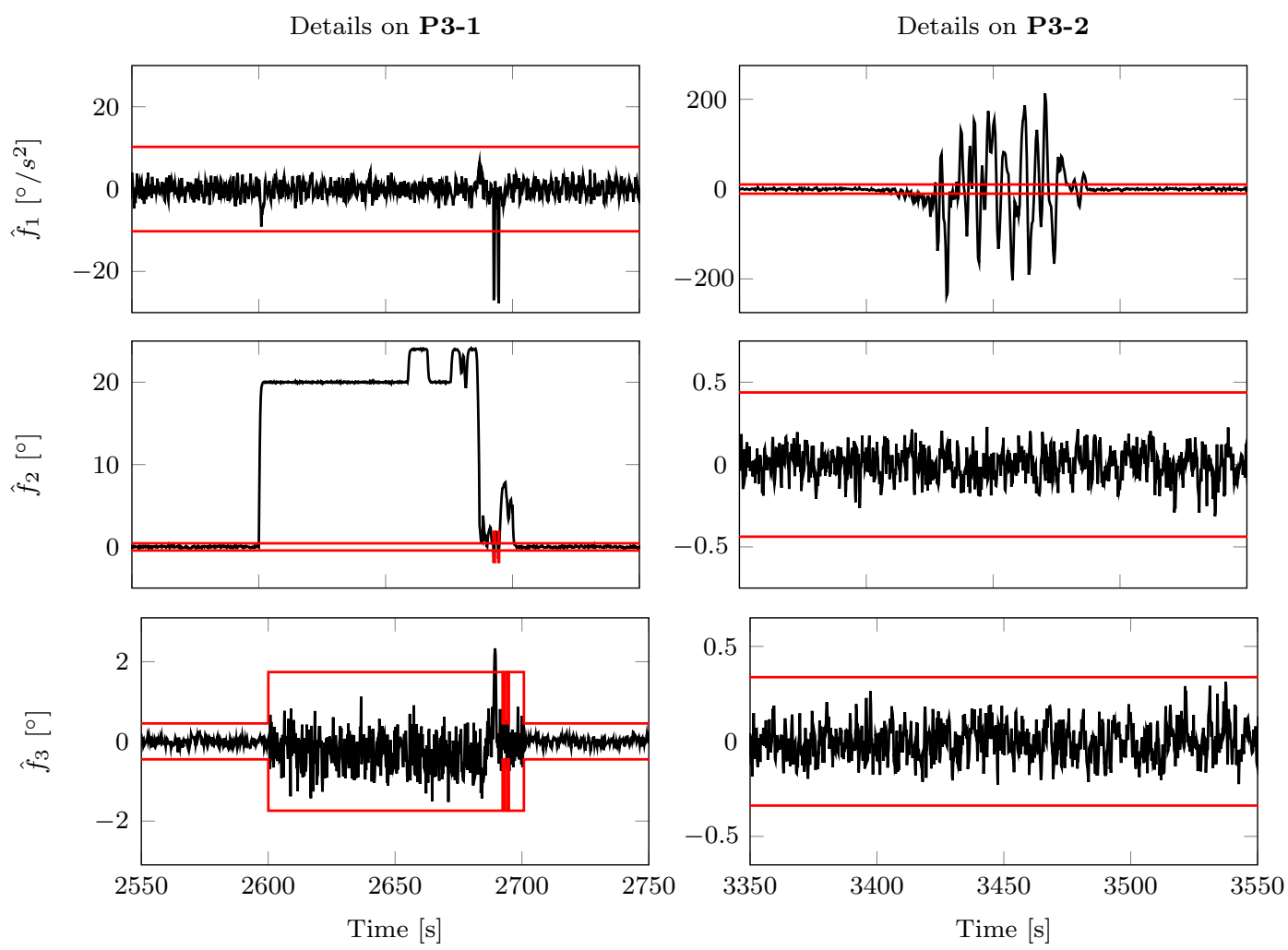

Figure 11: Details of FE and FI in the third pitch system (test set TS1).

\section{Conclusion}

In this work, we have developed performance-based designs of model-based observes and statistical-based decision mechanisms for achieving FE and FI in systems affected by unknown inputs and stochastic noises. First, we have presented FE performance-based designs of PI observers taking into account the trade-off between the degree of UI and interfault decoupling, the delay to track fault variations and the covariance due to noises. Second, we have presented FI performance-based co-designs of the observers and decision mechanisms taking into account the trade-off between the false isolation rates, the minimum isolable faults and the isolation times. Finally, we have extended the results to a scheme based on a bank of observers and decision mechanisms which provides a solution for FI and FE in systems where fault isolability conditions do not hold and it is not possible to achieve FE through standard observers. We have applied this procedure to a well-known benchmark that has 
explicit isolation requirements and we have shown that we fulfil all these requirements by just including them as constraints in the designs.

\section{Funding}

This work has been supported by the Spanish Ministry of Education, Culture and Sports (Grant no. FPU14/01592), the Spanish Ministry of Economy, Industry and Competitiveness (Project no. TEC2015-69155-R) and the Universitat Jaume I of Castelló (Project no. P11B2015-42).

\section{References}

Ahmadizadeh, S., Zarei, J., \& Karimi, H. R. (2014). Robust unknown input observer design for linear uncertain time delay systems with application to fault detection. Asian Journal of Control, 16(4), 1006-1019.

Aouaouda, S., Chadli, M., Shi, P., \& Karimi, H.-R. (2015). Discrete-time $H_{-} / H_{\infty}$ sensor fault detection observer design for nonlinear systems with parameter uncertainty. International Journal of Robust and Nonlinear Control, 25(3), 339-361.

Blesa, J., Jiménez, P., Rotondo, D., Nejjari, F., \& Puig, V. (2015). An interval NLPV parity equations approach for fault detection and isolation of a wind farm. IEEE Transactions on Industrial Electronics, 62(6), 37943805.

Blesa, J., Rotondo, D., Puig, V., \& Nejjari, F. (2014). FDI and FTC of wind turbines using the interval observer approach and virtual actuators/sensors. Control Engineering Practice, 24, 138-155.

Chang, J.-L. (2006). Applying discrete-time proportional integral observers for state and disturbance estimations. IEEE Transactions on Automatic Control, 51(5), 814-818.

Chen, J., \& Patton, R. J. (2012). Robust model-based fault diagnosis for dynamic systems (Vol. 3). Springer Science \& Business Media.

Chen, J., Patton, R. J., \& Zhang, H.-Y. (1996). Design of unknown input observers and robust fault detection filters. International Journal of Control, 63(1), 85-105.

Chen, L., Patton, R. J., \& Goupil, P. (2012). Robust fault estimation and performance evaluation based upon the ADDSAFE benchmark model. IFAC Proceedings Volumes, 45(20), 1364-1369.

Chen, W., Ding, S. X., Haghani, A., Naik, A., Khan, A. Q., \& Yin, S. (2011). Observer-based FDI schemes for wind turbine benchmark. In Proceedings of the ifac world congress, 2011 (pp. 7073-7078).

Chen, W., Khan, A., Abid, M., \& Ding, S. (2011). Integrated design of observer based fault detection for a class of uncertain nonlinear systems. International Journal of Applied Mathematics and Computer Science, 21(3), 423-430.

Cieslak, J., Efimov, D., \& Henry, D. (2015). Transient management of a supervisory fault-tolerant control scheme based on dwell-time conditions. International Journal of Adaptive Control and Signal Processing, 29(1), $123-142$.

de Oliveira, M. C., Bernussou, J., \& Geromel, J. C. (1999). A new discrete-time robust stability condition. Systems \& Control Letters, 37(4), 261-265.

Ding, S. X. (2008). Model-based fault diagnosis techniques: design schemes, algorithms, and tools. Springer Science \& Business Media.

Ding, S. X. (2014). Data-driven design of fault diagnosis and fault-tolerant control systems. Springer Science \& Business Media.

Dong, J., Wu, Y., \& Yang, G.-H. (2017). A new sensor fault isolation method for T-S fuzzy systems. IEEE Transactions on Cybernetics, 47(9), 2437-2447.

El Ghaoui, L., Oustry, F., \& AitRami, M. (1997). A cone complementarity linearization algorithm for static output-feedback and related problems. IEEE Transactions on Automatic Control, 42(8), 1171-1176.

Gao, Z. (2015). Fault estimation and fault-tolerant control for discrete-time dynamic systems. IEEE Transactions on Industrial Electronics, 62(6), 3874-3884. 
Gao, Z., Cecati, C., \& Ding, S. X. (2015). A survey of fault diagnosis and fault-tolerant techniques - Part I: Fault diagnosis with model-based and signal-based approaches. IEEE Transactions on Industrial Electronics, 62(6), 3757-3767.

Gao, Z., \& Ho, D. W. (2004). Proportional multiple-integral observer design for descriptor systems with measurement output disturbances. IEE Proceedings-Control Theory and Applications, 151(3), 279-288.

Gao, Z., Liu, X., \& Chen, M. Z. (2016). Unknown input observer-based robust fault estimation for systems corrupted by partially decoupled disturbances. IEEE Transactions on Industrial Electronics, 63(4), 25372547.

Guerra, T. M., Márquez, R., Kruszewski, A., \& Bernal, M. (2018). $H_{\infty}$ LMI-based observer design for nonlinear systems via Takagi-Sugeno models with unmeasured premise variables. IEEE Transactions on Fuzzy Systems, 26(3), 1498-1509.

Hassanabadi, A. H., Shafiee, M., \& Puig, V. (2016). UIO design for singular delayed LPV systems with application to actuator fault detection and isolation. International Journal of Systems Science, 47(1), 107121.

Henrion, D., Löfberg, J., Kočvara, M., \& Stingl, M. (2005). Solving polynomial static output feedback problems with PENBMI. In Proceedings of the 44th ieee conference on decision and control, 2005 (pp. 75817586).

Huang, S.-J., Zhang, D.-Q., Guo, L.-D., \& Wu, L.-B. (2018). Convergent fault estimation for linear systems with faults and disturbances. IEEE Transactions on Automatic Control, 63(3), 888-893.

Hwang, I., Kim, S., Kim, Y., \& Seah, C. E. (2010). A survey of fault detection, isolation, and reconfiguration methods. IEEE Transactions on Control Systems Technology, 18(3), 636-653.

Kočvara, M., \& Stingl, M. (2003). PENNON: A code for convex nonlinear and semidefinite programming. Optimization Methods and Software, 18(3), 317-333.

Lan, J., \& Patton, R. J. (2016). A new strategy for integration of fault estimation within fault-tolerant control. Automatica, 69, 48-59.

Lan, J., \& Patton, R. J. (2017). Integrated fault estimation and fault-tolerant control for uncertain lipschitz nonlinear systems. International Journal of Robust and Nonlinear Control, 27(5), 761-780.

Lan, J., Patton, R. J., \& Zhu, X. (2016). Fault-tolerant wind turbine pitch control using adaptive sliding mode estimation. Renewable Energy.

Li, X., Karimi, H. R., Wang, Y., Lu, D., \& Guo, S. (2018). Robust fault estimation and fault-tolerant control for markovian jump systems with general uncertain transition rates. Journal of the Franklin Institute, 355(8), $3508-3540$.

Li, X., \& Zhu, F. (2015). Simultaneous time-varying actuator and sensor fault reconstruction based on PI observer for LPV systems. International Journal of Adaptive Control and Signal Processing, 29(9), 1086-1098.

Li, X.-J., Yan, J.-J., \& Yang, G.-H. (2018). Adaptive fault estimation for TS fuzzy interconnected systems based on persistent excitation condition via reference signals. IEEE Transactions on Cybernetics(99), 1-13.

Li, Y., Karimi, H. R., Zhong, M., Ding, S. X., \& Liu, S. (2018). Fault detection for linear discrete time-varying systems with multiplicative noise: The finite-horizon case. IEEE Transactions on Circuits and Systems I: Regular Papers.

Li, Z., Mazars, E., Zhang, Z., \& Jaimoukha, I. M. (2012). State-space solution to the $H_{-} / H_{\infty}$ fault-detection problem. International Journal of Robust and Nonlinear Control, 22(3), 282-299.

Liu, M., \& Shi, P. (2013). Sensor fault estimation and tolerant control for itô stochastic systems with a descriptor sliding mode approach. Automatica, 49(5), 1242-1250.

Liu, X., Gao, Z., \& Chen, M. Z. (2017). Takagi-Sugeno fuzzy model based fault estimation and signal compensation with application to wind turbines. IEEE Transactions on Industrial Electronics, 64(7), 5678-5689.

Liu, X., Gao, Z., \& Zhang, A. (2018). Robust fault tolerant control for discrete-time dynamic systems with applications to aero engineering systems. IEEE Access, 6, 18832-18847.

Lofberg, J. (2004). YALMIP: A toolbox for modeling and optimization in MATLAB. In Proceedings of the ieee international symposium on computer aided control systems design, 2004 (pp. 284-289).

Odgaard, P. F., \& Stoustrup, J. (2012). Results of a wind turbine FDI competition. IFAC Proceedings Volumes, 45(20), 102-107. 
Odgaard, P. F., Stoustrup, J., \& Kinnaert, M. (2013). Fault-tolerant control of wind turbines: A benchmark model. IEEE Transactions on Control Systems Technology, 21(4), 1168-1182.

Odgaard, P. F., Stoustrup, J., Nielsen, R., \& Damgaard, C. (2009). Observer based detection of sensor faults in wind turbines. In Proceedings of the european wind energy conference, 2009 (pp. 4421-4430).

Pashazadeh, V., Salmasi, F. R., \& Araabi, B. N. (2018). Data driven sensor and actuator fault detection and isolation in wind turbine using classifier fusion. Renewable Energy, 116, 99-106.

Patton, R. J., \& Chen, J. (2000). On eigenstructure assignment for robust fault diagnosis. International Journal of Robust and Nonlinear Control, 10(14), 1193-1208.

Peñarrocha, I., Dolz, D., \& Sanchis, R. (2015). Performance trade-offs for networked jump observer-based fault diagnosis. IEEE Transactions on Signal Processing, 63(10), 2692-2703.

Rodrigues, M., Hamdi, H., Theilliol, D., Mechmeche, C., \& BenHadj Braiek, N. (2015). Actuator fault estimation based adaptive polytopic observer for a class of LPV descriptor systems. International Journal of Robust and Nonlinear Control, 25(5), 673-688.

Rotondo, D., Cristofaro, A., Johansen, T. A., Nejjari, F., \& Puig, V. (2016). Detection of icing and actuators faults in the longitudinal dynamics of small UAVs using an LPV proportional integral unknown input observer. In Proceedings of the 3rd conference on control and fault-tolerant systems (systol), 2016 (pp. 690-697).

Rotondo, D., Nejjari, F., Puig, V., \& Blesa, J. (2015). Model reference FTC for LPV systems using virtual actuators and set-membership fault estimation. International Journal of Robust and Nonlinear Control, 25(5), 735-760.

Salahshoor, K., Mosallaei, M., \& Bayat, M. (2008). Centralized and decentralized process and sensor fault monitoring using data fusion based on adaptive extended kalman filter algorithm. Measurement, 4l(10), 1059-1076.

Sales-Setién, E., Peñarrocha, I., Dolz, D., \& Sanchis, R. (2016). Performance-based design of PI observers for fault diagnosis in LTI systems under gaussian noises. In Proceedings of the 3rd conference on control and fault-tolerant systems (systol), 2016 (pp. 407-412).

Sales-Setién, E., \& Peñarrocha-Alós, I. (2018). Banks of estimators and decision mechanisms for pitch actuator and sensor FE in wind turbines. IFAC-PapersOnLine, 51(24), 1141-1148.

Sanchez, H., Escobet, T., Puig, V., \& Odgaard, P. F. (2015). Fault diagnosis of an advanced wind turbine benchmark using interval-based ARRs and observers. IEEE Transactions on Industrial Electronics, 62(6), 3783-3793.

Shi, F., \& Patton, R. (2015). An active fault tolerant control approach to an offshore wind turbine model. Renewable Energy, 75, 788-798.

Sloth, C., Esbensen, T., \& Stoustrup, J. (2011). Robust and fault-tolerant linear parameter-varying control of wind turbines. Mechatronics, 21(4), 645-659.

Wang, J., Ge, W., Zhou, J., Wu, H., \& Jin, Q. (2017). Fault isolation based on residual evaluation and contribution analysis. Journal of the Franklin Institute, 354(6), 2591-2612.

Wang, X., Tan, C. P., \& Zhou, D. (2017). A novel sliding mode observer for state and fault estimation in systems not satisfying matching and minimum phase conditions. Automatica, 79, 290-295.

Witczak, M., Buciakowski, M., Puig, V., Rotondo, D., \& Nejjari, F. (2016). An LMI approach to robust fault estimation for a class of nonlinear systems. International Journal of Robust and Nonlinear Control, 26(7), $1530-1548$.

Witczak, M., Rotondo, D., Puig, V., Nejjari, F., \& Pazera, M. (2017). Fault estimation of wind turbines using combined adaptive and parameter estimation schemes. International Journal of Adaptive Control and Signal Processing.

Wu, A.-G., \& Duan, G.-R. (2007). Generalized PI observer design for linear systems. IMA Journal of Mathematical Control and Information, 25(2), 239-250.

Wu, A.-G., Feng, G., \& Duan, G.-R. (2012). Proportional multiple-integral observer design for discrete-time descriptor linear systems. International Journal of Systems Science, 43(8), 1492-1503.

Yin, S., Gao, H., Qiu, J., \& Kaynak, O. (2017). Descriptor reduced-order sliding mode observers design for switched systems with sensor and actuator faults. Automatica, 76, 282-292. 
Zhang, K., Hao, H., Chen, Z., Ding, S. X., \& Peng, K. (2015). A comparison and evaluation of key performance indicator-based multivariate statistics process monitoring approaches. Journal of Process Control, 33, $112-126$.

Zhang, K., Jiang, B., \& Shi, P. (2012). Observer-based fault estimation and accomodation for dynamic systems (Vol. 436). Springer Science \& Business Media.

Zhang, P., \& Ding, S. X. (2008). An integrated trade-off design of observer based fault detection systems. Automatica, 44(7), 1886-1894.

Zhao, D., Shen, D., \& Wang, Y. (2017). Fault diagnosis and compensation for two-dimensional discrete time systems with sensor faults and time-varying delays. International Journal of Robust and Nonlinear Control, 27(16), 3296-3320.

Zhong, M., Zhang, L., Ding, S. X., \& Zhou, D. (2017). A probabilistic approach to robust fault detection for a class of nonlinear systems. IEEE Transactions on Industrial Electronics, 64(5), 3930-3939.

Zhou, K., Doyle, J. C., Glover, K., et al. (1996). Robust and optimal control (Vol. 40). Prentice hall New Jersey.

Ziyabari, S. H. S., \& Shoorehdeli, M. A. (2017). Robust fault diagnosis scheme in a class of nonlinear system based on UIO and fuzzy residual. International Journal of Control, Automation and Systems, 15(3), 11451154.

\section{A Proof of Theorem 1}

The following items prove each statement of Theorem 1 .

(i) Let us define the Lyapunov function $V^{Q}(k)=\tilde{z}(k)^{T} Q \tilde{z}(k)$ at each instant $k$. In the absence of UIs, noises and faults (i.e., $d=0, v=0, \delta=0$ ), after taking Schur's complements on (10) and premultiplying the result by $\left[\begin{array}{ll}\tilde{z}(k)^{T} & d(k)^{T}\end{array}\right]$ and postmultipliying by its transpose, we obtain that $V^{Q}(k+1)-V^{Q}(k) \leq$ 0 , which assures that the estimation error (6) converges to zero. We get the same result if we define the Lyapunov functions $V^{S}(k)=\tilde{z}(k)^{T} S \tilde{z}(k)$ and $V^{P_{i}}(k)=\tilde{z}(k)^{T} P_{i} \tilde{z}(k)$ at each instant $k$ and we perform similar steps on the first inequality in (11) and on (12) with $\left[\begin{array}{lll}\tilde{z}(k)^{T} & v(k)^{T}\end{array}\right]$ and $\left[\begin{array}{lll}\tilde{z}(k)^{T} & \delta(k)^{T}\end{array}\right]$, respectively.

(ii) In the absence of noises and faults (i.e., $v=0, \delta=0$ ), after taking Schur's complements on (10) and premultiplying the result by $\left[\tilde{z}(k)^{T} d(k)^{T}\right]$ and postmultipliying by its transpose, we obtain that

$$
V^{Q}(k+1)-V^{Q}(k)+\tilde{f}(k)^{T} \tilde{f}(k)-\gamma_{d} d(k)^{T} d(k) \leq 0 .
$$

Considering null initial conditions $\left(V^{Q}(0)=0\right)$ and adding the result from $k=0$ to $k=K-1$, it yields

$$
\sum_{k=0}^{K-1} \tilde{f}(k)^{T} \tilde{f}(k) \leq \sum_{k=0}^{K-1} \gamma_{d} d(k)^{T} d(k),
$$

where we have taken into account that $Q \succeq 0$. Dividing this expression by $K$ and taking the limit when $K \rightarrow \infty$, it leads to the second statement in Theorem 1 .

(iii) Applying a congruence transformation with $\left[\begin{array}{cc}S^{-1} & 0 \\ 0 & I\end{array}\right]$ to the first inequality in (11) and taking Schur's complements on the result lead to

$$
\overline{\mathcal{A}} S^{-1} \overline{\mathcal{A}}^{T}+\overline{\mathcal{G}} V \overline{\mathcal{G}}^{T} \preceq S^{-1} .
$$

In the absence of UIs and faults (i.e., $d=0, \delta=0$ ), the covariance of the estimation error $\tilde{z}, \Sigma^{z}=$ $\lim _{k \rightarrow \infty} \mathbb{E}\left\{\tilde{z}(k) \tilde{f}(z)^{T}\right\}$, fulfils the Lyapunov equation (9a). Then, we deduce that $\Sigma^{z} \preceq S^{-1}$ because $\overline{\mathcal{A}}$ has stable eigenvalues as demonstrated in the first item of this proof. Applying Schur's complements on the second inequality in (11) we have that

$$
\overline{\mathcal{R}} S^{-1} \overline{\mathcal{R}}^{T}+K \mathcal{H} V(K \mathcal{H})^{T} \preceq \Xi .
$$


In the absence of UIs and faults (i.e., $d=0, \delta=0$ ), the covariance of the estimation error $\tilde{f}, \Sigma=$ $\lim _{k \rightarrow \infty} \mathbb{E}\left\{\tilde{f}(k) \tilde{f}(k)^{T}\right\}$, fulfils the Lyapunov equation (9b) and we deduce that $\Sigma \preceq \Xi$.

(iv) In the absence of UIs and noises (i.e., $d=0, v=0$ ), multiplying (12) with $\left[\tilde{z}(k)^{T} \quad \delta(k)^{T}\right]$ on the left and by its transpose on the right and performing similar steps as in the second statement, we prove the fourth statement in Theorem 1.

\section{B State-space Matrices of the Wind Turbine Model}

The state-space matrices of the converter model are $A^{c}=-\alpha_{g c}$ and $B^{c}=\alpha_{g c}$. The matrices $C^{c}, F^{c}$ and $H^{c}$ equal the identity matrix of appropriate dimensions while $E^{c}, G^{c}$ and $D^{c}$ are zero.

The state-space matrices of the drive train model are

$$
\begin{gathered}
A^{c}=\left[\begin{array}{ccc}
a_{11} & a_{12} & a_{13} \\
a_{21} & a_{22} & a_{23} \\
1 & a_{32} & 0
\end{array}\right], B^{c}=\left[\begin{array}{l}
0 \\
b \\
0
\end{array}\right], G^{c}=\left[\begin{array}{ccccc}
0 & 0 & 0 & 0 & 0 \\
0 & 0 & 0 & 0 & -b \\
0 & 0 & 0 & 0 & 0
\end{array}\right], D^{c}=\left[\begin{array}{c}
1 / J_{r} \\
0 \\
0
\end{array}\right] \\
C^{c}=\left[\begin{array}{lll}
1 & 0 & 0 \\
1 & 0 & 0 \\
0 & 1 & 0 \\
0 & 1 & 0
\end{array}\right], H^{c}=\left[\begin{array}{lllll}
1 & 0 & 0 & 0 & 0 \\
0 & 1 & 0 & 0 & 0 \\
0 & 0 & 1 & 0 & 0 \\
0 & 0 & 0 & 1 & 0
\end{array}\right]
\end{gathered}
$$

with $a_{11}=\frac{-\left(B_{d t}+B_{r}\right)}{J_{r}}, a_{12}=\frac{B_{d t}}{N_{g} J_{r}}, a_{13}=\frac{-K_{d t}}{J_{r}}, a_{21}=\frac{\eta_{d t, 0} B_{d t}}{N_{g} J_{g}}, a_{32}=\frac{-1}{N_{g}}, a_{22}=\frac{-\left(\eta_{d t, 0} B_{d t}+B_{g} N_{g}^{2}\right)}{N_{g}^{2} J_{g}}$, $a_{23}=\frac{\eta_{d t, 0} K_{d t}}{N_{g} J_{g}}$ and $b=\frac{-1}{J_{g}}$. We have that $E^{c}=0$ and $F^{c}=I$.

The state-space matrices of each pitch model are

$$
\begin{gathered}
A^{c}=\left[\begin{array}{cc}
0 & 1 \\
2 c & 2 w_{n_{0}} \xi_{0}
\end{array}\right], B^{c}=\left[\begin{array}{c}
0 \\
-2 c
\end{array}\right], E^{c}=\left[\begin{array}{lll}
0 & 0 & 0 \\
1 & c & c
\end{array}\right], G^{c}=\left[\begin{array}{ll}
0 & 0 \\
c & c
\end{array}\right] \\
C^{c}=\left[\begin{array}{ll}
1 & 0 \\
1 & 0
\end{array}\right], F^{c}=\left[\begin{array}{lll}
0 & 1 & 0 \\
0 & 0 & 1
\end{array}\right]
\end{gathered}
$$

with $c=-w_{n_{0}}^{2} / 2, H^{c}=I$ and $D^{c}=0$. 